\title{
Effect of Reynolds number on turbulent drag reduction by superhydrophobic surface textures
}

\author{
Jin Lee • Thomas O. Jelly • Tamer A. Zaki
}

Received: date / Accepted: date

\begin{abstract}
In turbulent flows over streamwise-aligned superhydrophobic surface (SHS) textures, the percent drag reduction is dependent on Reynolds number. This dependence is examined using direct numerical simulations of channel flow over SHS texture at three bulk Reynolds numbers, $R e_{b}=2800,6785$ and 10975 . Simulations of regular no-slip channel flows at the same bulk velocities are also performed for comparison. Changes in the flow due to the SHS texture are examined with particular focus on phase averaged statistics and coherent turbulent motions. As the Reynolds number is increased, the texture performance, or the percent drag reduction, is enhanced. In terms of turbulent motions, the weakened Jin Lee

Department of Mechanical Engineering, Johns Hopkins University, Baltimore, MD 21218, USA

Thomas O. Jelly

Department of Engineering, Imperial College London, South Kensington Campus, London SW7 2AZ, UK

Tamer A. Zaki $(\bowtie)$

Department of Mechanical Engineering, Johns Hopkins University, Baltimore, MD 21218, USA

E-mail: t.zaki@jhu.edu
\end{abstract}


shear stresses are manifest as a decrease in the population of the small-scale vortical structures and a weakening of large-scale motions. The influence of stochastic fluctuations near the large-scale motions diminishes at higher Reynolds number. The enhanced performance is therefore due to a significant drop in the strength of small- and large-scale vortical structures. The weaker vortical motions yield a considerable reduction in the Reynolds shear stress and the transport of mean momentum. The SHS texture introduces a performance penalty in the form of a local increase in drag at the no-slip free-slip edge. This adverse effect is caused by the inhomogeneity of the near-wall flow field. However, the affected region narrows in width at higher Reynolds numbers. As a result, the percent drag reduction approaches the gas fraction of the texture.

Keywords Turbulence $\cdot$ drag reduction $\cdot$ channel flows $\cdot$ DNS 


\section{Introduction}

Superhydrophobicity is achieved through the combination of small-scale surface features and a hydrophobic chemical coating. Contrary to a no-slip surface, a superhydrophobic surface (SHS) immersed in a liquid can trap gas-bubbles between surface protrusions to form a gas-liquid interface, which corresponds to the CassieBaxter state [1]. When the liquid is in motion, preservation of the interface enables the flow to slip along a shear-free region supported between surface features, which results in significant reductions in skin-friction drag compared to a regular no-slip flow.

SHS drag reduction in turbulent channel flow has been verified experimentally for a range of surface textures. Daniello et al. [2] reported that turbulent drag reduction begins as the dimensions of the SHS features and viscous sublayer thickness become commensurate. The texture performance increases with wider feature spacing and at higher Reynolds number $(R e)$, provided that the surface does not transition to the Wenzel state [3]. Woolford et al. [4] collected turbulence statistics above a streamwise-aligned SHS, and reported a decrease in the streamwise turbulence intensity, Reynolds shear stress and turbulent kinetic energy production, relative to a regular no-slip configuration. Aljallis et al. [5] recently demonstrated that the benefits of superhydrophobicity can be lost at high Reynolds numbers due to depletion of the gas bubbles trapped between SHS features. Developments of hierarchical roughness [6] and bubble-restoring surfaces [7] are aimed at addressing this issue and present promising strategies to maximize the turbulent drag-reducing properties of SHS textures. 
Motivated by the analytical work by Philip [8], a model that represents SHS features and interfaces as flat regions of no-slip and free-slip boundary conditions, respectively, has been adopted in a number of computational studies. Martell et al. [9] performed direct numerical simulation (DNS) of turbulent channel flow past a number of SHS ridge and post geometries at a fixed friction Reynolds number, $R e_{\tau}=180$, and reported reductions in the wall shear stress of up to $40 \%$. Their findings were in agreement with the experimental results by Daniello et al. [2] for a SHS ridge geometry with $30 \mu \mathrm{m}$ spacing. In a subsequent study, Martell et al. [10] quantified the SHS performance at three fixed friction Reynolds numbers, $R e_{\tau}=180,395$ and 590, and noted that SHS performance exhibited a Reynolds number dependence when scaled by outer units. However, they remarked that a more universal behavior in turbulent drag reduction may exist by choosing an appropriate inner scaling.

The analysis of streamwise-aligned SHS textures bears similarity to the study of longitudinal riblets [11], where the spanwise inhomogeneity alters the base flow. Türk et al. [12] and Jelly et al. [13] reported phase-averaged statistics from DNS of turbulent channel flow over streamwise aligned SHS textures, and demonstrated that a secondary flow of Prandtl's second kind was established at the no-slipfree-slip boundary. The secondary flow is manifest as coherent counter-rotating streamwise vortices. Jelly et al. [13] examined the primary, secondary and turbulent fluid motions in detail and their contributions to skin friction. The performance of SHS texture was shown to be adversely affected by spanwise diffusion of the primary flow and the additional streamwise-spanwise stress, $\left\langle R_{13}\right\rangle$, which is absent in the regular no-slip channel flow. On the other hand, the secondary flow and the reduction in the Reynolds shear stress, $\left\langle R_{12}\right\rangle$, both improved performance. 
By systematically varying the feature width and spacing and also the Reynolds number, a series of DNS by Park et al. [14] characterized SHS performance in both laminar and turbulent channel flows. Laminar drag reduction was found to be independent of Reynolds number and was determined by the texture geometry alone - an observation in agreement with several theoretical investigations [1517]. Park et al. [14] demonstrated that the reductions in turbulent drag collapsed onto a single curve when expressed as a function of the effective slip length in wall units. They also noted that for maximum turbulent drag reduction to be achieved, the effective slip length expressed in wall units must approach a value equivalent to the height of the buffer layer. Furthermore, a maximum turbulent drag reduction of $50 \%$ was reported for streamwise-aligned ridges with an equal feature size and spacing beyond 200 non-dimensional wall units. These findings are of practical importance, and motivate a close examination of the mechanism of drag reduction, its dependence on the Reynolds number, and the associated changes in turbulence statistics and structures.

The present study investigates the mechanism of drag reduction in turbulent channel flow over SHS texture at three bulk Reynolds numbers, $R e_{b}=2800,6875$ and 10975. Regular no-slip channel flows at the same bulk Reynolds numbers are also simulated for comparison. In addition to the phase-averaged statistics, vortical structures and coherent patterns of large-scale motions are examined to explain the changes in the flow statistics due to the SHS texture, and the improved performance at higher Re. This paper is organized into five sections. Section 2 describes the computational approach, phase-averaging procedure and validation. Section 3 details the effect of the SHS texture on the flow statistics. Section 4 reports changes in the turbulence structures in the vicinity of the texture. Changes 
in both the small and large-scale motions reinforce the findings based on the statistical response. Finally, a summary is presented in Section 5.

\section{Numerical methodology}

\subsection{Governing equations}

The continuity and the Navier-Stokes equations for an incompressible flow are,

$$
\begin{gathered}
\frac{\partial u_{j}}{\partial x_{j}}=0 \\
\frac{\partial u_{i}}{\partial t}+u_{j} \frac{\partial u_{i}}{\partial x_{j}}=-\frac{\partial p}{\partial x_{i}}+\frac{1}{R e_{b}} \frac{\partial^{2} u_{i}}{\partial x_{j} \partial x_{j}}
\end{gathered}
$$

In equations (1-2), the Cartesian velocity components, $u_{i}=(u, v, w)$, are aligned along their respective coordinates, $x_{i}=(x, y, z)$, and $p$ denotes the pressure. All variables are non-dimensionalized by the channel half-height, $\delta$, and the bulk velocity, $U_{b}$. The bulk Reynolds number is therefore $R e_{b} \equiv U_{b} \delta / \nu$, where $\nu$ is the kinematic viscosity. In addition to the outer scaling, the viscous scaling is introduced using the friction velocity $u_{\tau} \equiv \sqrt{\left(\tau_{w} / \rho\right)}$ where $\tau_{w}=\left.\mu \frac{\partial\langle U\rangle}{\partial y}\right|_{w}$ and subscript ' $w$ ' indicates that the quantity is evaluated at the wall. Hereinafter, the symbol $u_{\tau 0}$ denotes the friction velocity of a regular no-slip channel flow, which has the same bulk Reynolds number. Using $u_{\tau 0}$, the friction Reynolds number is $R e_{\tau 0} \equiv u_{\tau 0} \delta / \nu$. Quantities non-dimensionalized by wall units are identified by superscript + , for example $y^{+}=y u_{\tau 0} / \nu$.

A fractional step algorithm is adopted to advance the momentum equation (equation 2). The convective, diffusive and pressure terms are treated using explicit Adams-Bashforth, implicit Crank-Nicolson and implicit Euler schemes, respectively. The spatial derivatives in equations (1-2) are evaluated using a second- 

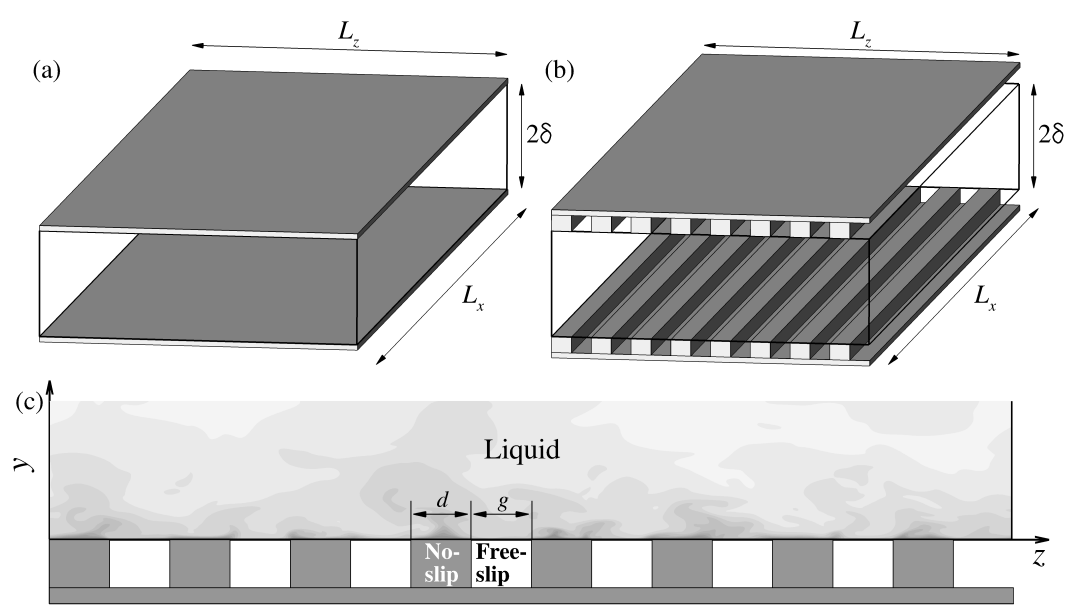

Fig. 1 Schematic of computational domains for the (a) regular no-slip and (b) SHS configurations. (c) In the SHS case, no-slip and free-slip boundary conditions are enforced to model the micro-ridges and interfaces, respectively.

order accurate finite volume method, where the field variables are stored in a staggered arrangement. The current algorithm has been used extensively in DNS of transitional $[19,20]$ and fully turbulent boundary-layers $[21,22]$, and of turbulent channel flow with streamwise-aligned SHS textures [13].

\subsection{Boundary conditions and statistical averaging}

The computational domain is depicted in figure 1 . The rectangular volume $\left(L_{x} \times L_{y} \times L_{z}\right)$ is discretized using a Cartesian mesh with $\left(N_{x} \times N_{y} \times N_{z}\right)$ grid points. Uniform mesh spacing is adopted in the streamwise and spanwise directions. In the wallnormal direction, grid points are clustered near the walls using a hyperbolic tangent stretching function.

The SHS is characterized by streamwise-aligned micro-ridges of width $d$ and liquid-gas interfaces of width $g$. The tops of the features and the interfaces are 
represented using a flat surface with alternating no-slip and free-slip boundary conditions (figure 1c). The mixed no-slip/free-slip model has been adopted in a number of recent DNS in order to examine turbulent drag reduction using SHS textures (e.g. $[12,13])$. Contrary to the previous simulation setup by Martell et al. $[9,10]$, the SHS texture is prescribed on both the upper and lower walls (figure 1b) in order to maintain statistical symmetry about the channel centerline.

For phase-averaging, a spanwise phase is defined using the modulo operator 'mod',

$$
\phi_{z} \equiv 2 \pi\left(\frac{z}{d+g} \bmod 1\right)
$$

and therefore $\phi_{z}$ is in the range $0 \leq \phi_{z}<2 \pi$. An instantaneous field variable, $f$, can be decomposed as

$$
\begin{aligned}
f(\mathbf{x}, t) & =\underbrace{\langle F(y)\rangle^{x z}+\tilde{f}\left(y, \phi_{z}\right)}_{\langle f\rangle}+f^{\prime}(\mathbf{x}, t) \\
& =\left\langle f\left(y, \phi_{z}\right)\right\rangle+f^{\prime}(\mathbf{x}, t)
\end{aligned}
$$

where $f^{\prime}$ is the fluctuating component and $\langle\cdot\rangle$ is the time and phase average [18]. The notation $\langle\cdot\rangle^{x z}$ indicates further averaging in the streamwise and spanwise directions (note that upper case variables also denote $\langle\cdot\rangle^{x z}$, e.g. $U_{i}=\left\langle u_{i}\right\rangle^{x z}$ ). The periodic component can be recovered according to $\tilde{f}\left(y, \phi_{z}\right)=\left\langle f\left(y, \phi_{z}\right)\right\rangle-$ $\langle F(y)\rangle^{x z}$. In addition, when $d=g$ the instantaneous velocities can be decomposed as

$$
\begin{aligned}
& u_{i}(\mathbf{x}, t)=\quad\left\langle u_{i}\right\rangle\left(y, \phi_{z}\right)+u_{i}^{\prime}(\mathbf{x}, t) \\
& =\left\langle U_{i}\right\rangle^{x z}(y)+\tilde{u}_{i}\left(y, \phi_{z}\right) \quad+u_{i}^{\prime}(\mathbf{x}, t) \\
& =\frac{1}{2}\left(\left\langle U_{i}\right\rangle^{\mathrm{ns}}(y)+\left\langle U_{i}\right\rangle^{\mathrm{fs}}(y)\right)+\tilde{u}_{i}\left(y, \phi_{z}\right)+u_{i}^{\prime}(\mathbf{x}, t),
\end{aligned}
$$


Table 1 Simulation parameters.

\begin{tabular}{cccccccccc}
\hline Case & $R e_{b}$ & $L_{x}$ & $L_{z}$ & $N_{x}$ & $N_{y}$ & $N_{z}$ & $\Delta x^{+}$ & $\Delta y_{\min }^{+}$ & $\Delta z^{+}$ \\
\hline L-REF and L-SHS & 2800 & 12 & 3 & 512 & 128 & 1024 & 3.75 & 0.19 & 0.52 \\
M-REF and M-SHS & 6785 & 9 & 3 & 576 & 384 & 1536 & 6.17 & 0.16 & 0.76 \\
H-REF and H-SHS & 10975 & 9 & 3 & 768 & 512 & 2048 & 6.91 & 0.15 & 0.86 \\
\hline
\end{tabular}
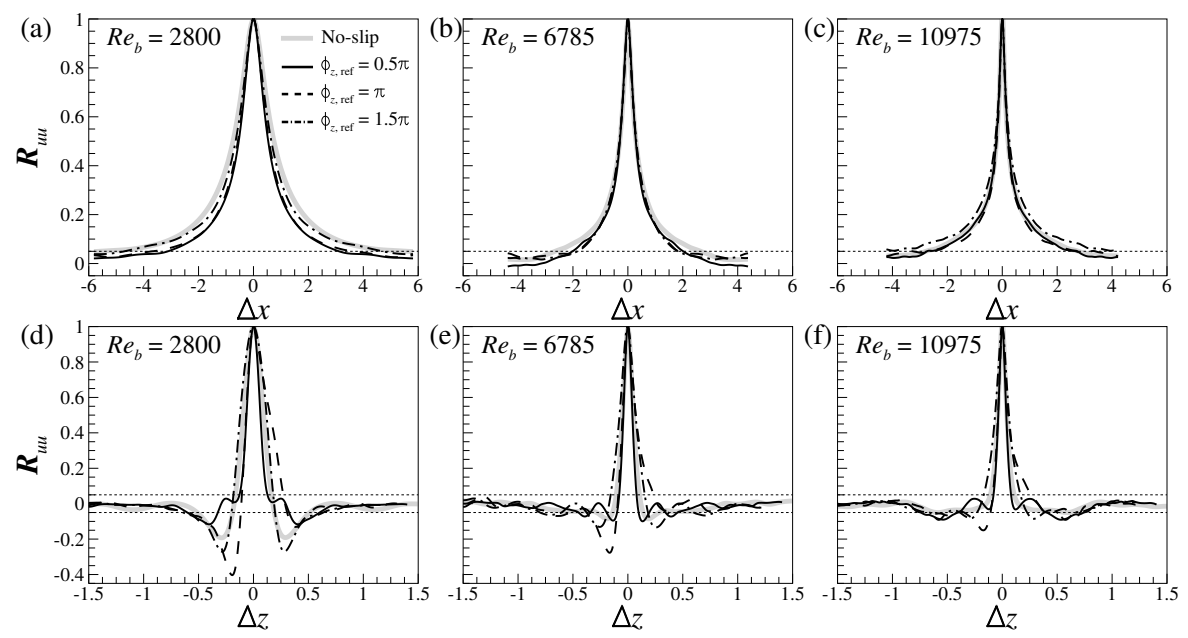

Fig. 2 (a-c) streamwise and (d-f) spanwise two-point correlations of streamwise velocity fluctuations, $\boldsymbol{R}_{u u}$, at $y=0.1 \delta$. (a,d) $R e_{b}=2800$; (b,e) $R e_{b}=6785 ;$ (c,f) $R e_{b}=10975$. Horizontal dotted lines mark $\boldsymbol{R}_{u u}= \pm 0.05$.

where superscripts 'ns' and 'fs' identify averages over all no-slip and free-slip phases, respectively (see figure 1c).

\subsection{Validation and computational setup}

Details of the grid resolution and dimensions of the computational domain adopted throughout this study are provided in table 1 . The first letter in the designation of each case refers to the Reynolds numbers: "L" is the low- $\left(R e_{b}=2800\right.$ and $\left.R e_{\tau 0}=180\right)$; "M" is the moderate- $\left(R e_{b}=6785\right.$ and $\left.R e_{\tau 0}=395\right)$; and "H" is the 
high-Reynolds-number flow $\left(R e_{b}=10975\right.$ and $\left.R e_{\tau 0}=590\right)$. The subsequent three letters refer to the boundary conditions: "REF" is the reference simulation with no-slip boundaries; and "SHS" is the superhydrophobic configuration. Throughout, the width of the no-slip and the free-slip regions are fixed, $d=g=0.1875$. This spacing is equivalent to the $30-30 \mu \mathrm{m}$ ridges examined by Martell et al. [10]. Although the size of the SHS texture is equal among all cases in outer scaling, it varies when expressed in wall units. The length of the computational domain in the streamwise direction is longer than previous studies (e.g., Martell et al. [10] and Park et al. [14]) in order to minimize the influence of the streamwise periodic boundary conditions. In the spanwise direction, the domain is the same width as in the simulations by Martell et al. [10]. As shown in figure 2, two-point correlations of the streamwise velocity fluctuations are below $5 \%$ of the maximum value at the end of the computational domain. To ensure that the flow is sufficiently resolved in the spanwise direction, a grid spacing $\Delta z^{+}<1$ is adopted in all the simulations, which is finer than previous simulations of the same configuration (see table 1). The requirement that $\Delta z^{+}<1$ is based on the grid refinement study performed by Jelly et al. [13], where extensive validation was also reported. The computational time step is $\Delta t=4 \times 10^{-3}$ for the low $R e$ and $\Delta t=1 \times 10^{-3}$ for the moderate and high $R e$ cases. These values correspond to $\Delta t^{+}<5 \times 10^{-2}$ in all cases. Timeand phase-averaged statistics were collected during 221 convective time units in order to ensure convergence of the phase-averaged and conditional statistics.

The mean streamwise velocity profiles and Reynolds stresses, $\left\langle R_{i j}\right\rangle \equiv\left\langle u_{i}^{\prime} u_{j}^{\prime}\right\rangle$, are shown in figures 3 and 4, respectively. The results from the regular no-slip simulations (figures 3(i) and 4(i)) are in good agreement with the data by Moser et al. [23]. Note that the grid resolution of their data $\left(\Delta x^{+}=9.7-17.7\right.$ and $\Delta z^{+}=$ 

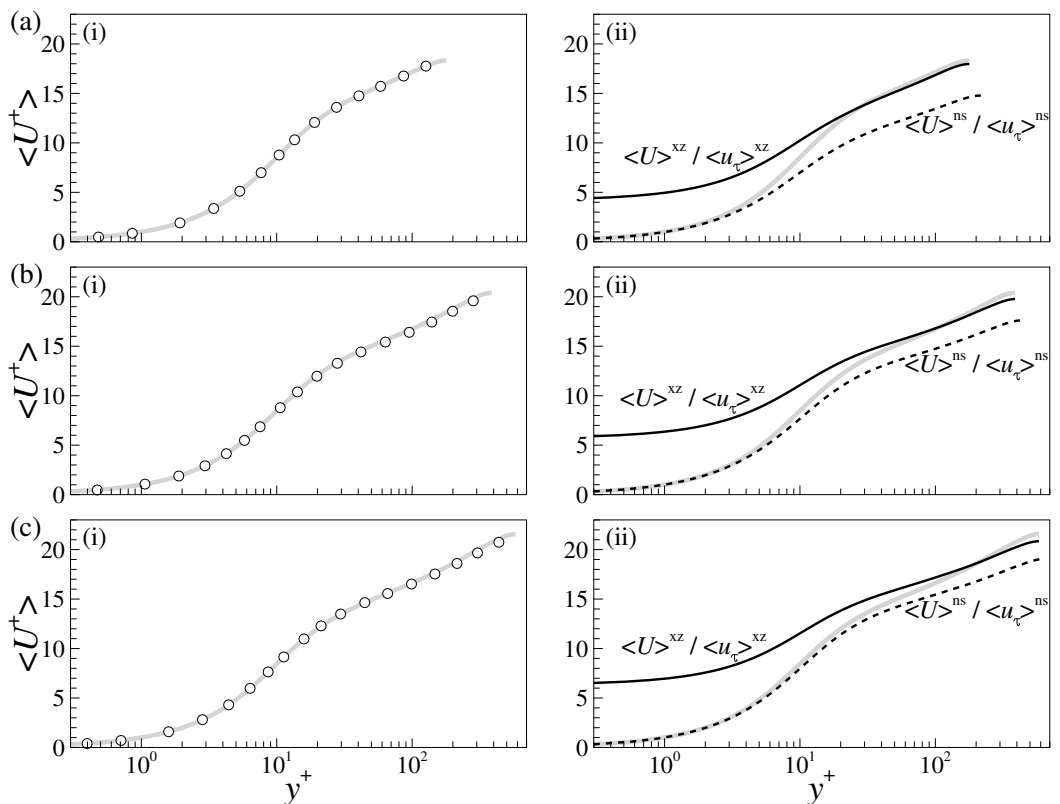

Fig. 3 Mean streamwise velocity for (a) $R e_{b}=2800$, (b) $R e_{b}=6785$ and (c) $R e_{b}=10975$. Gray and black lines represent the regular channel and SHS flows, and the dashed lines are $\langle U\rangle^{n s} /\left\langle u_{\tau}\right\rangle^{n s}$. Results by Moser et al. [23] are plotted as symbols.

$5.9-4.8)$ is larger than in the current study, since they focused on the regular noslip channel flow without surface inhomogeneities. The mean streamwise velocity profiles of the SHS cases are plotted in figure 3(ii). The profiles of $\langle U\rangle^{x z} /\left\langle u_{\tau}\right\rangle^{x z}$ are shifted upward in the near-wall region due to the slip velocity. In order to maintain the same bulk velocity as the regular no-slip flow, the mean velocity decreases at the channel core. Streamwise and spanwise normal stresses of the SHS cases (figure 4(ii)) are large in the viscous sublayer due to the slip condition, while all shown components of the Reynolds stresses are weakened in the buffer and outer regions relative to the regular no-slip channel flow. Figure 4(ii) also reports the stresses on the no-slip phases, $\left\langle R_{i j}\right\rangle^{n s} /\left\langle u_{\tau}^{2}\right\rangle^{n s}$. While their near-wall 

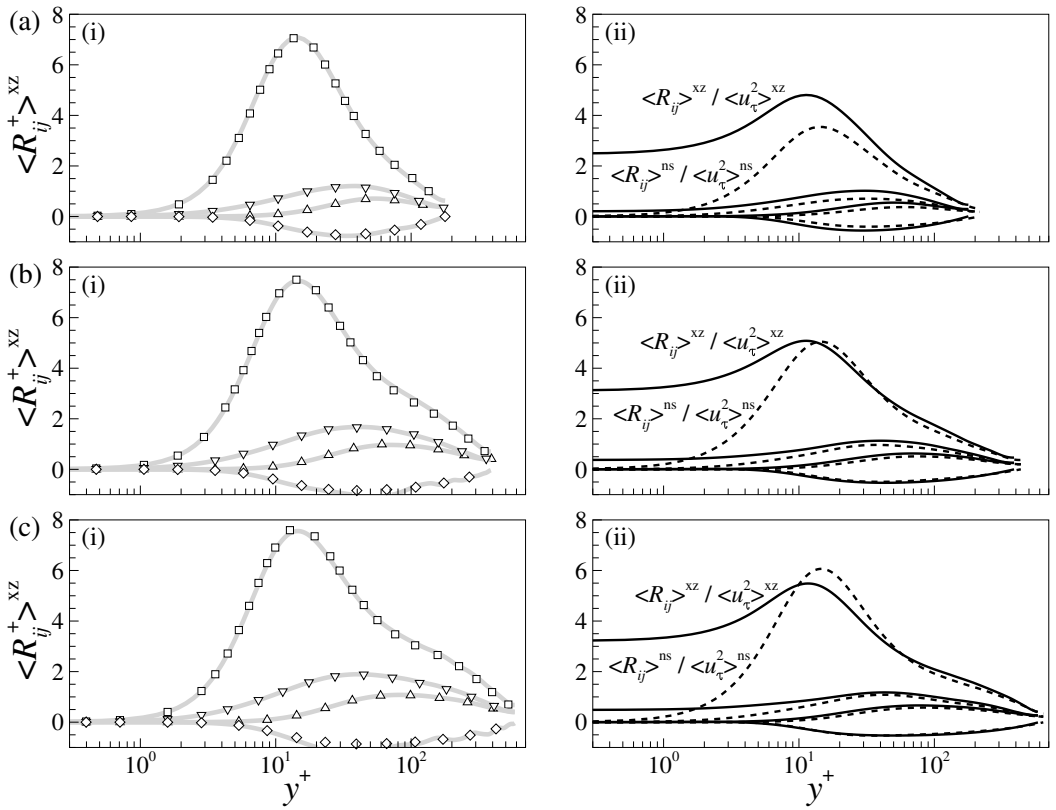

Fig. 4 Reynolds stress profiles for (a) $R e_{b}=2800$, (b) $R e_{b}=6785$ and (c) $R e_{b}=10975$. Gray and black lines represent the regular and SHS channel flows, respectively. Results by Moser et al. [23] are plotted as symbols. $(\square)\left\langle R_{11}\right\rangle ;(\triangle)\left\langle R_{22}\right\rangle ;(\nabla)\left\langle R_{33}\right\rangle ;(\diamond)\left\langle R_{12}\right\rangle$.

behavior resembles the reference no-slip flow, they reach weaker levels in the buffer and outer regions.

\section{Statistical analysis}

Integral performance parameters are reported in table 2 . The skin-friction coefficient $C_{f}\left(\equiv \tau_{w} / \frac{1}{2} \rho U_{b}^{2}\right)$ on the SHS texture is substantially lower than in the regular channel. As the Reynolds number increases, the mean streamwise slip velocity, $\left\langle U_{s}\right\rangle^{x z}$, increases and drag reduction is enhanced. The performance trend is therefore consistent with previous studies $[9,10]$. In outer scaling, the slip length, $\left\langle l_{u}\right\rangle^{x z}\left(\equiv\left\langle U_{s}\right\rangle^{x z} /\left.\frac{\partial\langle U\rangle^{x z}}{\partial y}\right|_{w}\right)$, is reduced at higher Reynolds number due to the increase in the mean-shear rate. Park et al. [14] reported that performance sat- 
Table 2 Integral performance parameters: Skin-friction coefficient $\left(C_{f}\right)$, slip velocity $\left(U_{s}\right)$, slip length $\left(l_{u}\right)$ and drag-reduction rate $(D R)$.

\begin{tabular}{cccccc}
\hline Case & $\left\langle C_{f}\right\rangle_{0}^{x z} \times 10^{3}$ & $\left\langle C_{f}\right\rangle^{x z} \times 10^{3}$ & $\left\langle U_{s}\right\rangle^{x z}$ & $\left\langle l_{u}\right\rangle^{x z}$ & $D R$ \\
\hline L-SHS & 8.10 & 6.35 & 0.270 & 0.0301 & 21.6 \\
M-SHS & 6.51 & 4.26 & 0.323 & 0.0222 & 34.6 \\
H-SHS & 5.66 & 3.53 & 0.337 & 0.0174 & 37.7 \\
\hline
\end{tabular}

urated at a drag-reduction rate $D R=50 \%$ when the feature spacing $(d+g)$ exceeds 200 wall units (figure 5), where $D R \equiv\left(1-\left\langle C_{f}\right\rangle^{x z} /\left\langle C_{f}\right\rangle_{0}^{x z}\right) \times 100$. While this condition is satisfied for case H-SHS, the recorded $D R$ is $37.7 \%$ only. The difference in performance relative to the work by Park et al. [14] is due to the difference in boundary conditions. Park et al. [14] prescribed SHS with streamwise slip only, and in the current study we allow both streamwise and spanwise slip. The spanwise slip, in general, has a detrimental impact on drag reduction [24-26]. It is nonetheless clear that performance as measured by the drag reduction rate is improved at higher $R e$. The associated changes in the base flow and turbulence and their influence on skin friction are examined below.

\subsection{Primary and secondary mean flows}

As shown in figure 3(ii), the mean streamwise velocity averaged across all phases, $\langle U\rangle^{x z} /\left\langle u_{\tau}\right\rangle^{x z}$, progressively departs from the log-law with increasing Reynolds number. This departure from the log-law is symptomatic of the improved SHS performance at higher Reynolds numbers. On the other hand, the streamwise velocity profiles averaged across no-slip phases only are in good agreement with the linear law in the viscous sublayer. The velocity profiles $\langle U\rangle^{n s} /\left\langle u_{\tau}\right\rangle^{n s}$ are, 


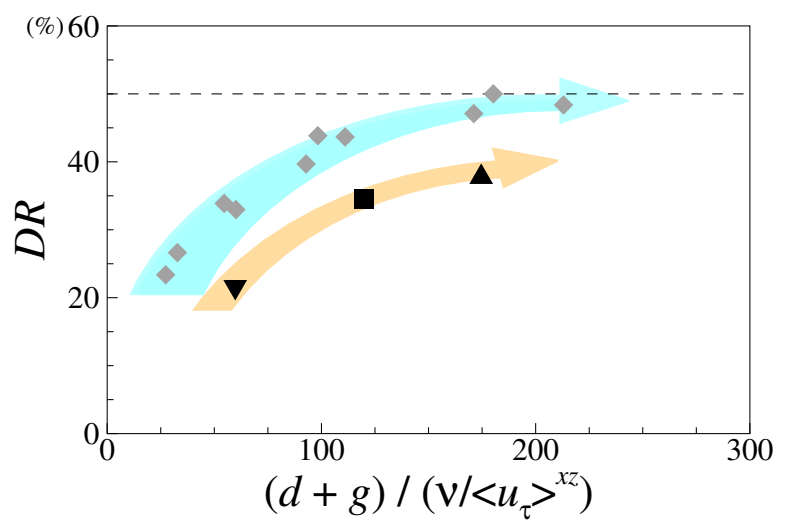

Fig. 5 Drag-reduction rate $(D R)$ as a function of pitch $(d+g)$ normalized by inner-length scale $\left(\nu /\left\langle u_{\tau}\right\rangle^{x z}\right)$. Two-dimensional slip in $x$ and $z$ (present results): $\mathbf{\nabla}$, L-SHS; $\mathbf{\square}$, M-SHS; H-SHS. Results for one dimensional slip in $x$ are adopted from Park et al. [14].

however, shifted downward in the buffer and log-law regions - a behavior which suggests drag increase on the no-slip phases. As the Reynolds number increases, the departure from the regular no-slip profile in the log-law region is reduced.

Contours of the primary flow in the $\left(\phi_{z}-y\right)$ plane are shown in figures 6(i) and (ii). A qualitatively similar primary flow pattern is observed for all Reynolds numbers: A boundary layer forms above the no-slip (NS) region, flanked by fastmoving fluid that slips along the adjoining free-slip (FS) regions. Vectors of $(\tilde{v}, \tilde{w})$ are also plotted on the $\left(\phi_{z}-y\right)$ plane in figure 6(iii). The number of vectors has been reduced for clarity. A pair of streamwise vortices is observed at the NS-FS edge in the phase-averaged flow field. They cause an upwash above the NS surface and a downwash on the FS boundary. The origin of the secondary flow was explained by Türk et al.[12] and Jelly et al.[13]. The source of the streamwise vorticity in the secondary flow is predominantly $\mathcal{P}=-\frac{\partial}{\partial z}\left(\frac{\partial}{\partial y}\left\langle R_{33}\right\rangle\right)$ [13], which is also plotted in figure 6(ii) and increases at the NS-FS edge with increasing Reynolds number. 

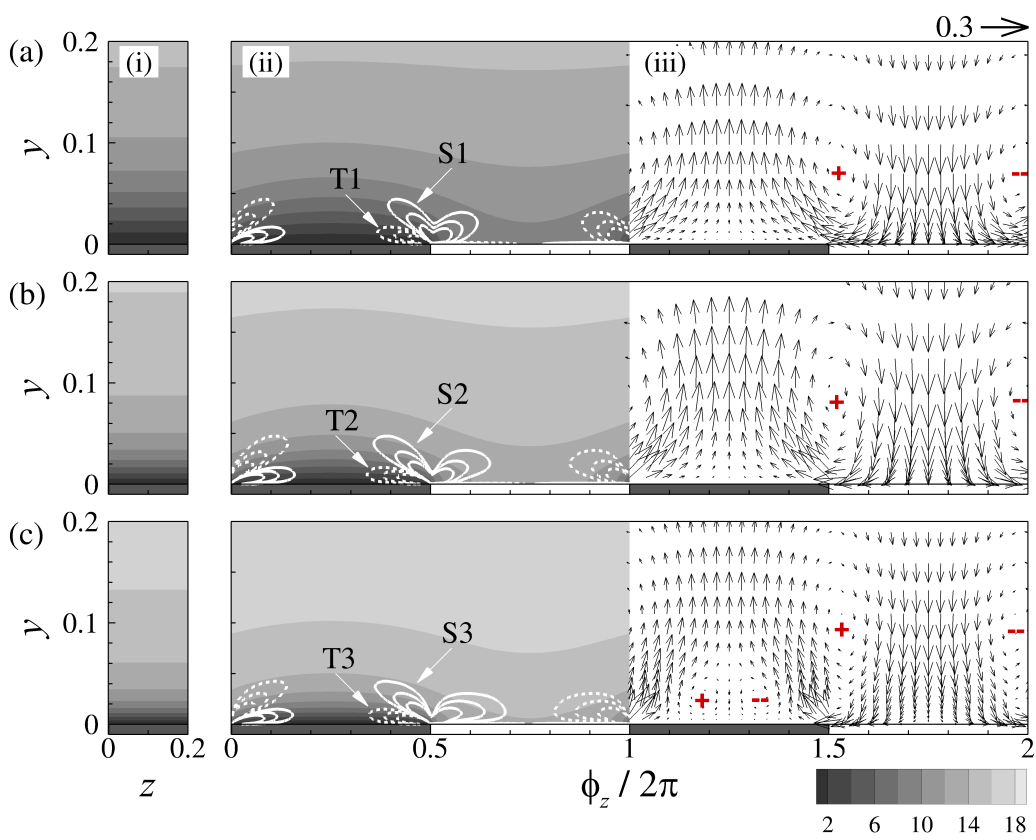

Fig. 6 Time- and phase-averaged streamwise velocity (flood), source term $\mathcal{P}$ of streamwise vorticity (lines; $\{-2,-1,-0.5$ (dashed) $0.5,1,2$ (solid) $\})$ and in-plane velocity vectors $\left(\tilde{v}^{+}, \tilde{w}^{+}\right)$. (a) $R e_{b}=2800$, (b) $R e_{b}=6785$ and (c) $R e_{b}=10975$. (i) Regular channel, (ii-iii) SHS. Symbols '十' and '-' mark the centers of the clockwise and counterclockwise rotating vortex cores.

The larger area of the $\mathcal{P}$-contour at higher $R e$ (marked 'S1', 'S2' and 'S3') is consistent with a stronger secondary flow, or streamwise vorticity. The spanwise position of the vortex cores is similar in all cases. The secondary flow is such that it transports high-momentum fluid in the spanwise direction from the free-slip region towards the no-slip wall. This pattern is followed by the upwash above the central no-slip phases. In the highest-Reynolds-number flow, a very weak tertiary flow is observed in the central region of the no-slip surface. The associated $\mathcal{P}$ (marked 'T1', 'T2' and 'T3') has opposite sign to the larger adjacent secondary flow ('S1', 'S2' and 'S3'). The emergence of tertiary flow at higher Re opposes the action of 

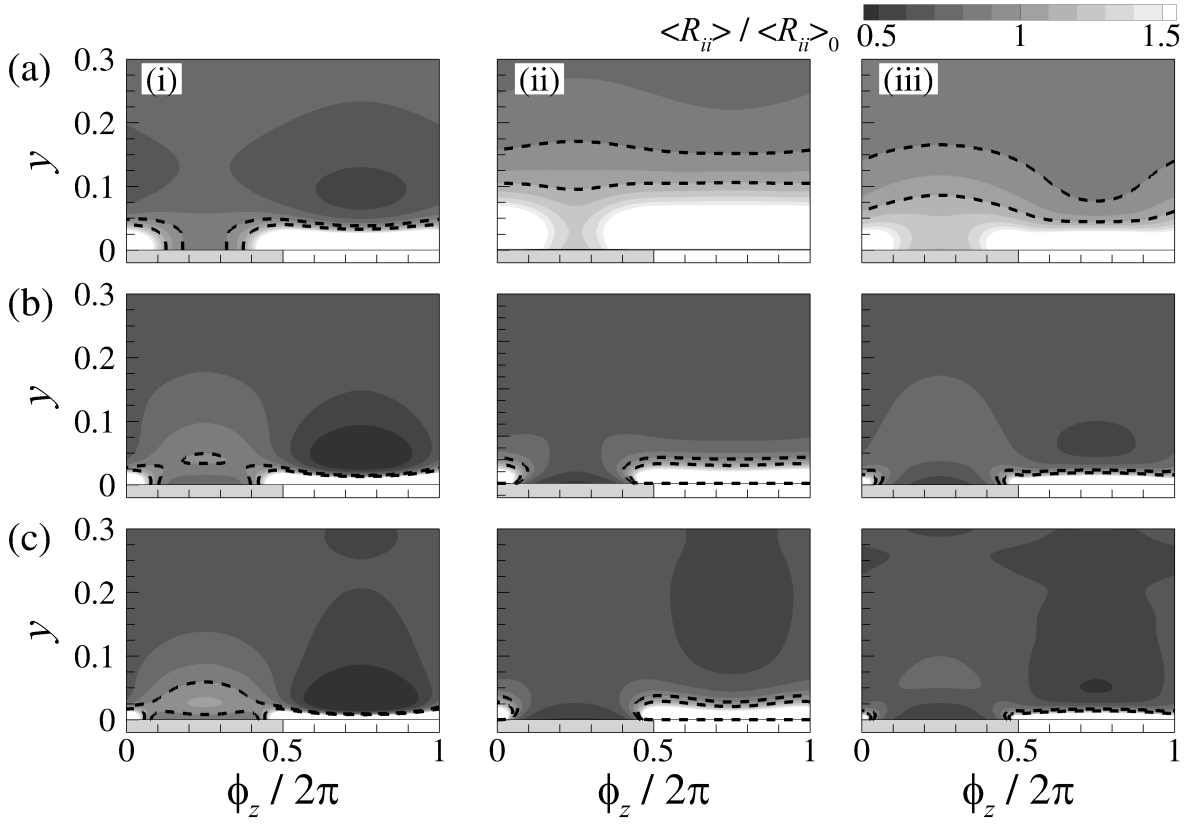

Fig. 7 Ratio of the Reynolds normal stresses in the SHS case to the regular channel. (a) LSHS, (b) M-SHS and (c) H-SHS. (i) streamwise $\left\langle R_{11}\right\rangle /\left\langle R_{11}\right\rangle_{0}$, (ii) wall-normal $\left\langle R_{22}\right\rangle /\left\langle R_{22}\right\rangle_{0}$ and (iii) spanwise $\left\langle R_{33}\right\rangle /\left\langle R_{33}\right\rangle_{0}$ components. Dashed line contours mark the levels 0.9 and 1.1.

the secondary flow and weakens the transport of the high-momentum fluid from the FS to the NS region.

\subsection{Reynolds stresses}

In addition to the changes in the mean flow, turbulent motions are also strongly influenced by the SHS texture. Figure 7 shows the ratios of the Reynolds normal stresses in the flow over the SHS to those in regular channel flow. All components of the normal stress are weakened in the outer region. The spanwise dependence of the isocontours is contained within the region $y<(d+g)$. At low $R e$, the edge 

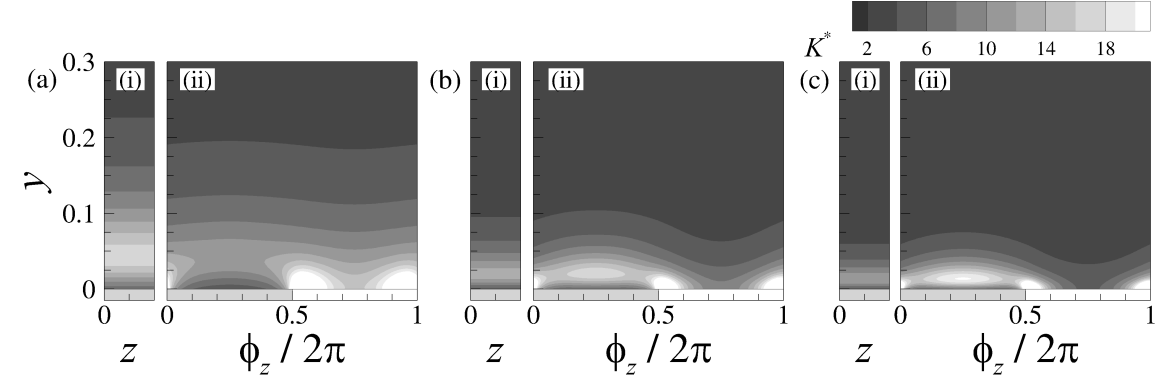

Fig. 8 Energy partition parameter $K^{*}$ for (a) $R e_{b}=2800$, (b) $R e_{b}=6785$ and (c) $R e_{b}=$ 10975. (i) Regular channel and (ii) the SHS channel flows.

effect of the texture, where the normal stresses are increased, persists towards the central phases of the no-slip region. At high Re, the middle of the texture is less influenced by the edges and the stresses resemble those in regular channel flow. The energy partition parameter, $K^{*} \equiv\left(2\left\langle R_{11}\right\rangle\right) /\left(\left\langle R_{22}\right\rangle\left\langle R_{33}\right\rangle\right)$, is shown in figure 8. Its large value in the NS region relative to the regular channel flow indicates higher level of anisotropy. The change in isotropy from the FS to the NS region indicates that the turbulence structures differ between the two zones.

The Reynolds shear stresses have a direct influence on skin friction [27]. In addition to $\left\langle R_{12}\right\rangle=\left\langle u^{\prime} v^{\prime}\right\rangle$, the spanwise inhomogeneity of the mean flow introduces a streamwise-spanwise component $\left\langle R_{13}\right\rangle$. The ratio of the Reynolds shear stress, $\left\langle R_{12}\right\rangle /\left\langle R_{12}\right\rangle_{0}$, is plotted in figure 9(i). Relative to the regular channel flow, $\left\langle R_{12}\right\rangle$ is weakened over the no-slip region of the SHS texture. Note that a region of positive $\left\langle R_{12}\right\rangle$ (or negative $\left\langle R_{12}\right\rangle /\left\langle R_{12}\right\rangle_{0}$ ) is observed at the NS-FS edge and leads to a negative contribution to TKE production. Its origin was explained by Jelly et al. [13] and its peak value increases with Re. The other two components, $\left\langle R_{23}\right\rangle^{+}$and $\left\langle R_{13}\right\rangle^{+}$which vanish in the regular channel, are finite in the SHS flow. Note that, in regular channel flows, $v^{\prime}$ and $w^{\prime}\left(u^{\prime}\right.$ and $\left.w^{\prime}\right)$ are spatially cor- 

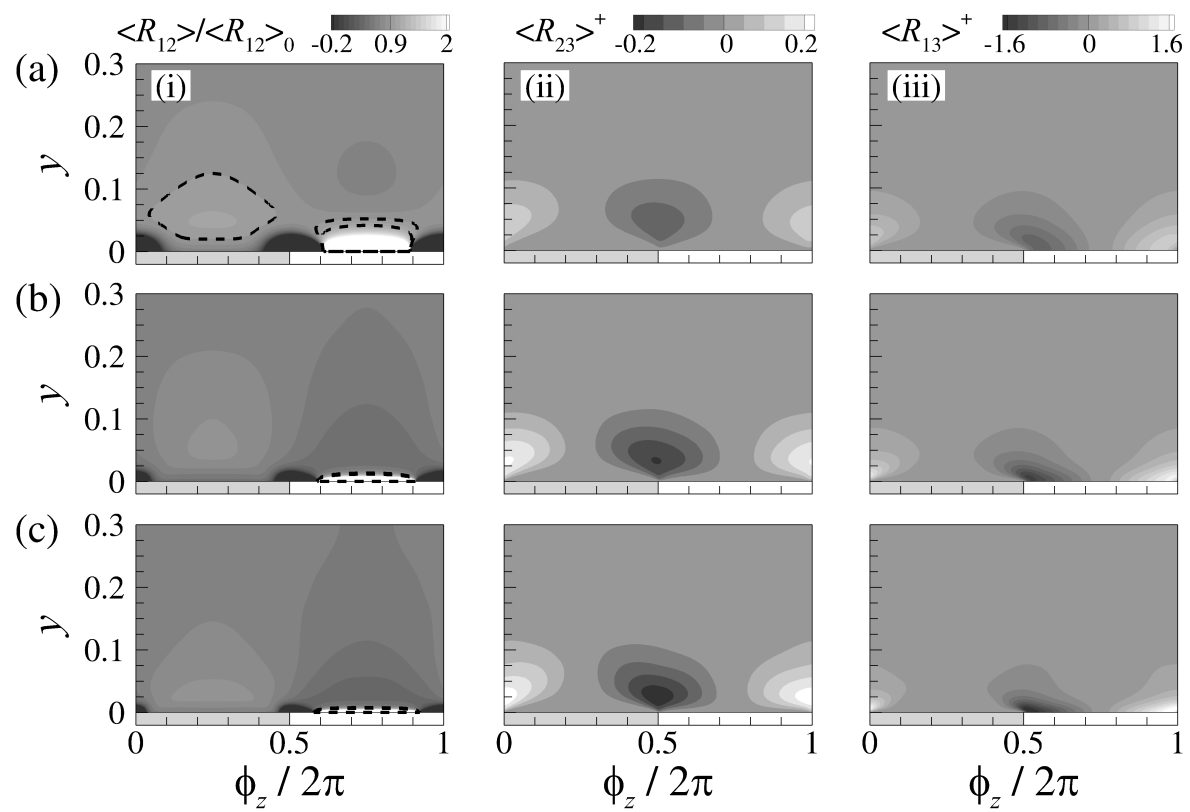

Fig. 9 (i) Ratio of streamwise-wall-normal stress in the SHS case to the regular channel, $\left\langle R_{12}\right\rangle /\left\langle R_{12}\right\rangle_{0}$. Dashed line contours mark the levels 0.9 and 1.1. (ii) wall-normal-spanwise $\left\langle R_{23}\right\rangle$ and (iii) streamwise-spanwise $\left\langle R_{13}\right\rangle$ stresses. (a) L-SHS, (b) M-SHS and (c) H-SHS.

related around streamwise vortices (wall-normal vortices), but these correlations are counterbalanced by the randomness of the associated flow structures in space. In terms of magnitude, peak values of $\left\langle R_{23}\right\rangle^{+}$and $\left\langle R_{13}\right\rangle^{+}$are doubled when $R e_{b}$ increases from 2800 to 10975 . Because the peak is located at the NS-FS edge, it results in a very large gradient of the shear stresses at that location. The finite values of $\left\langle R_{13}\right\rangle^{+}$and $\left\langle R_{23}\right\rangle^{+}$due to the inhomogeneous boundary conditions in the span constitute a qualitative change in the turbulence over SHS textures.

Quantitatively, the reduction in turbulence kinetic energy in the buffer and outer regions is appreciable, and is attributed to the free-slip phases of the texture (figure 7). On the other hand, the no-slip phases support a local increase in $\left\langle R_{i i}\right\rangle$ 

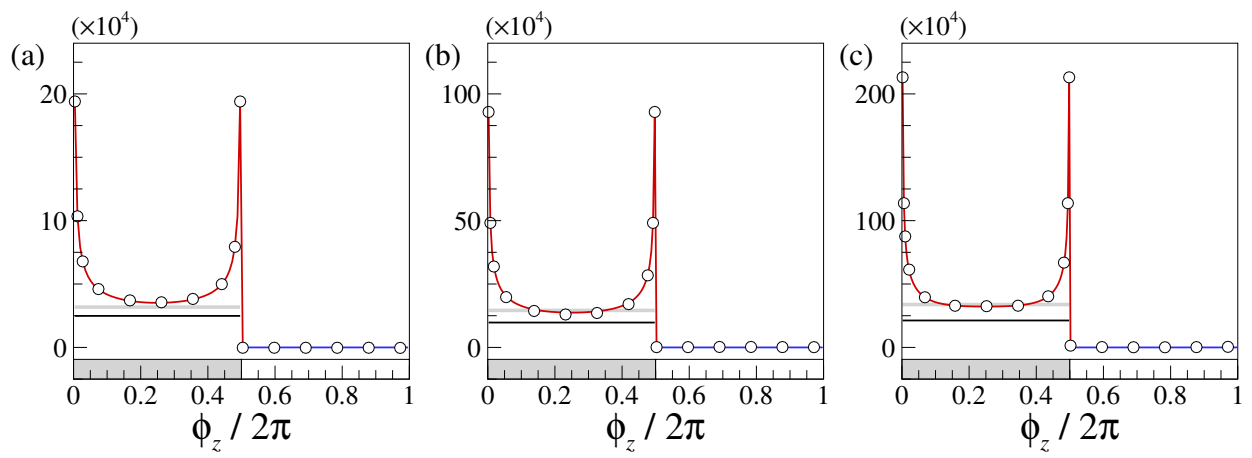

Fig. 10 Squared friction Reynolds number $R e_{\tau}^{2}$ in: (red) no-slip region; (blue) free-slip region; (gray) regular channel flow; (black) The average $\left\langle R e_{\tau}^{2}\right\rangle^{x z}$; (symbols) $\left\langle R e_{\tau}^{2}\right\rangle$ from the identity (6). (a) L-SHS, (b) M-SHS and (c) H-SHS

especially near their periphery. In general, with a reduced turbulence activity, there is an anticipated drag reduction [21]. In order to clarify the contribution of the mean and turbulent quantities to the drag, a detailed analysis of the skin-friction coefficient is presented in the next subsection.

\subsection{Skin-friction budget}

The drag-reduction rate $(D R)$ is not solely determined by the ratio of the no-slip and free-slip regions, although $D R$ approaches the gas fraction, $g /(d+g)$, at large $R e$ (figure 5). Previously, Jelly et al. [13] investigated SHS channel flow at $R e_{\tau 0}=$ 180 and reported that the local skin-friction coefficient $C_{f}(z)$ in the NS region is greater than that of regular channel flow. Specifically, $C_{f}$ has an overshoot at the NS-FS edge. In order to explain the variation of the skin friction, we examine the contribution of various statistical turbulence quantities as a function of spanwise phase and $R e$. 
The contributions of primary, secondary and turbulent fluid motions to the skin-friction coefficient $C_{f}$ are quantified using a phase-averaged extension of the identity first introduced by Fukagata et al. [27]. In order to compare different Reynolds numbers with the same velocity scale, the identity is derived for the friction Reynolds number,

$$
\begin{aligned}
& \left\langle R e_{\tau}^{2}\right\rangle_{\text {FIK }=} \overbrace{3 R e_{b}\left(1-\left\langle U_{s}\right\rangle\right)}^{\text {Laminar contribution, }\left\langle R e_{\tau}^{2}\right\rangle_{\text {lam }}^{2}} \overbrace{+\frac{3 R e_{b}}{2} \int_{0}^{1}(1-y)^{2} \frac{\partial^{2}}{\partial \overline{\langle u\rangle}}}^{\text {Spanwise diffusion, }\left\langle R e_{\tau}^{2}\right\rangle_{\text {zdff }}} d y \\
& \underbrace{-\frac{3 R e_{b}^{2}}{2} \int_{0}^{1}(1-y)^{2}\left(\overline{\overline{\tilde{v} \frac{\partial\langle u\rangle}{\partial y}}}\right) d y}_{\text {Wall-normal advection, }\left\langle R e_{\tau}^{2}\right\rangle_{\tilde{v}}} \underbrace{-\frac{3 R e_{b}^{2}}{2} \int_{0}^{1}(1-y)^{2}\left(\overline{\bar{w} \frac{\partial\langle u\rangle}{\partial z}}\right) d y}_{\text {Spanwise advection, }\left\langle R e_{\tau}^{2}\right\rangle_{\tilde{w}}} \\
& +\underbrace{3 R e_{b}^{2} \int_{0}^{1}(1-y)\left\langle-R_{12}\right\rangle d y}_{\left\langle R_{12}\right\rangle \text { contribution, }\left\langle R e_{\tau}^{2}\right\rangle_{R_{12}}} \underbrace{+\frac{3 R e_{b}^{2}}{2} \int_{0}^{1}(1-y)^{2}\left(-\frac{\partial \overline{\overline{\left\langle R_{13}\right\rangle}}}{\partial z}\right) d y}_{\left\langle R_{13}\right\rangle \text { contribution, }\left\langle R e_{\tau}^{2}\right\rangle_{R_{13}}}
\end{aligned}
$$

where $\overline{\overline{\langle\cdot\rangle}}=\langle\cdot\rangle-\int_{0}^{1}\langle\cdot\rangle \mathrm{d} y$. As shown in figure 10, the friction Reynolds number obtained from the identity perfectly matches the definition $R e_{\tau} \equiv \frac{u_{\tau} \delta}{\nu}$. Moreover, this figure demonstrates that, despite of overall reduction in skin friction, the friction Reynolds number over the no-slip phases exceeds its reference value from the regular channel simulation. Although $\left\langle R e_{\tau}^{2}\right\rangle_{\text {FIK }}$ on the central no-slip phases nearly matches the value from the regular channel flow, it has a sharp peak at the texture edge in all cases. The presence of the large positive peak limits the overall drag reduction.

The various contributions to $\left\langle R e_{\tau}^{2}\right\rangle$ in the identity (equation 6) are reported in figure 11. Note that regular channel flows only have the laminar $\left\langle R e_{\tau}^{2}\right\rangle_{\text {lam }}$ and the Reynolds shear stress $\left\langle R e_{\tau}^{2}\right\rangle_{R 12}$ contributions [27]. All additional terms are 

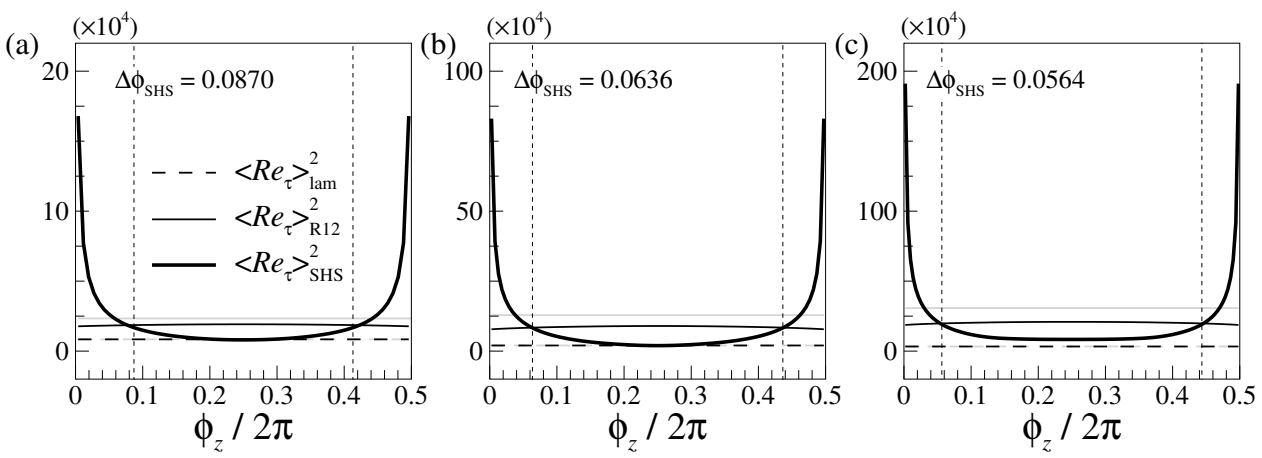

Fig. 11 Decomposition of the friction Reynolds number over the no-slip phases, $0<\phi_{z} / 2 \pi<$ 0.5. (a) L-SHS, (b) M-SHS and (c) H-SHS. Black, the SHS channel; gray, regular channel.

lumped into $\left\langle R e_{\tau}^{2}\right\rangle_{\mathrm{SHS}}$,

$$
\left\langle R e_{\tau}^{2}\right\rangle_{\mathrm{FIK}}=\left\langle R e_{\tau}^{2}\right\rangle_{\mathrm{lam}}+\left\langle R e_{\tau}^{2}\right\rangle_{\mathrm{R} 12}+\left\langle R e_{\tau}^{2}\right\rangle_{\mathrm{SHS}}
$$

Only the no-slip phases are considered in figure 11 since drag is identically zero on the free-slip surface. The contribution by $\left\langle R_{12}\right\rangle$ in the SHS channel is smaller than in regular channel flow, and the difference is more pronounced at higher Reynolds number. The term, $\left\langle R e_{\tau}^{2}\right\rangle_{\mathrm{SHS}}$ is most significant near the edge between the NS and FS regions. Also note that the edge region $\Delta \phi_{\mathrm{SHS}}$, defined as the spanwise width to reach $10 \%$ of the peak value, narrows at larger $R e$ (see vertical dashed lines in figure 11). At a given $R e$, the net effect of the reduction in the contribution by $\left\langle R_{12}\right\rangle^{n s}$ and the increase in $\left\langle R e_{\tau}^{2}\right\rangle_{\text {SHS }}^{n s}$ is a larger value of $\left\langle R e_{\tau}^{2}\right\rangle^{n s}$ compared to a regular channel, although the normalized value of $\left\langle R e_{\tau}^{2}\right\rangle^{n s} / R e_{\tau 0}^{2}$ tends to unity and hence $\left\langle R e_{\tau}^{2}\right\rangle^{x z} / R e_{\tau 0}^{2}$ tends to 0.5 .

Figure 12 decomposes $\left\langle R e_{\tau}^{2}\right\rangle_{\mathrm{SHS}}$. into its three constituents. As shown in the figure, $\left\langle R e_{\tau}^{2}\right\rangle_{\text {zdiff }}$ has a large value near the edges. The peak value is nearly two orders of magnitude higher than at the center of the NS region in the H-SHS case, which demonstrates the origin of the sharp increase in $\left\langle R e_{\tau}^{2}\right\rangle_{\mathrm{SHS}}$ at the NS-FS 

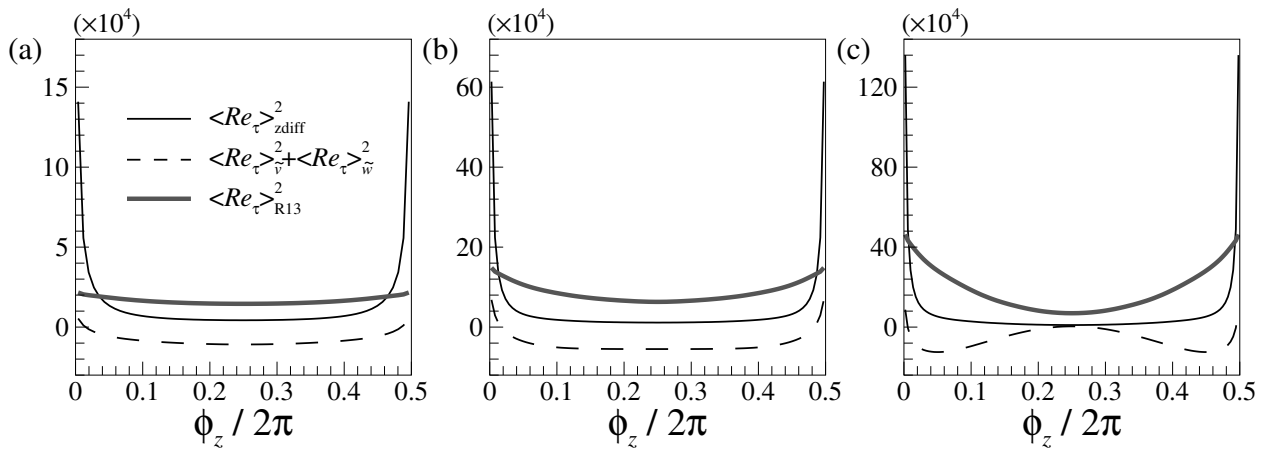

Fig. 12 Decomposition of the SHS terms in the friction Reynolds number, $\left\langle R e_{\tau}^{2}\right\rangle_{\mathrm{SHS}}$ as a function of phase, $0<\phi_{z} / 2 \pi<0.5$. (a) L-SHS, (b) M-SHS and (c) H-SHS.

edge (figure 11). As the Reynolds number increases, the influence of the texture edges is confined to a narrow region where the spanwise diffusion is dominant. Similarly, $\left\langle R e_{\tau}^{2}\right\rangle_{\mathrm{R} 13}$ contributes an increase in skin friction (adverse effect on drag reduction). This term corresponds to turbulent diffusion in the spanwise direction. Although the maximum value of the $\left\langle R e_{\tau}^{2}\right\rangle_{\mathrm{R} 13}$ profile is smaller than $\left\langle R e_{\tau}^{2}\right\rangle_{\mathrm{zdiff}}$, the former term has a considerable magnitude over a wider region. Recall that $\left|\left\langle R_{13}\right\rangle^{+}\right|$in the H-SHS case significantly increases at the NS-FS edge (figure 9). This concentrated region of large $\left|\left\langle R_{13}\right\rangle^{+}\right|$leads to a large drag because this term appears as a spanwise gradient in the equation for $\left\langle R e_{\tau}^{2}\right\rangle$. The present results reinforce the recent finding that turbulent diffusion reduces slip effects $[12,13]$.

The secondary flow contributes to the skin friction through two terms, $\left\langle R e_{\tau}^{2}\right\rangle_{\tilde{v}}$ and $\left\langle R e_{\tau}^{2}\right\rangle_{\tilde{w}}$ [13]. Their net contribution, $\left\langle R e_{\tau}^{2}\right\rangle_{\tilde{v}}^{n s}+\left\langle R e_{\tau}^{2}\right\rangle_{\tilde{w}}^{n s}$, generally acts as a reduction of drag. The sign of $\left\langle R e_{\tau}^{2}\right\rangle_{\tilde{v}}+\left\langle R e_{\tau}^{2}\right\rangle_{\tilde{w}}$ is opposite to $\left\langle R e_{\tau}^{2}\right\rangle_{\mathrm{R} 13}$ near the central no-slip phase $\left(\phi_{z} / 2 \pi=0.25\right)$, where these terms nearly balance. As a result, the most significant contributions to $\left\langle R e_{\tau}^{2}\right\rangle$ are $\left\langle R e_{\tau}^{2}\right\rangle_{\text {zdiff }}$ and $\left\langle R e_{\tau}^{2}\right\rangle_{\mathrm{R} 13}$ near the NS-FS edge. At higher Reynolds number, the spanwise region of influence 
of these terms, or the edge effect, is reduced. If we denote this edge region $\delta_{e}$ and the averaged skin-friction coefficient in $\delta_{e}$ as $\left\langle C_{f}\right\rangle^{\delta_{e}}$, then the influence of $\delta_{e}$ on $D R$ can be approximated by,

$$
D R / 100 \equiv 1-\frac{\left\langle C_{f}\right\rangle^{x z}}{\left\langle C_{f}\right\rangle_{0}^{x z}} \approx 1-\left(\frac{d-\delta_{e}}{d+g}+\frac{\left\langle C_{f}\right\rangle^{\delta_{e}}}{\left\langle C_{f}\right\rangle_{0}^{x z}} \frac{\delta_{e}}{d+g}\right) .
$$

The drag reduction rate approaches the gas fraction as $\delta_{e} /(d+g)$ tends to zero. This can be achieved by using wider textures or at higher $R e$ where $\delta_{e}$ is reduced, provided that the increase in $\frac{\left\langle C_{f}\right\rangle^{\delta_{e}}}{\left\langle C_{f}\right\rangle_{0}^{x z}}$ is slower than the decrease in $\delta_{e} /(d+g)$.

In summary, even though $\left\langle R e_{\tau}^{2}\right\rangle^{n s}>R e_{\tau 0}^{2}$ over the NS region, the ratio $\left\langle R e_{\tau}^{2}\right\rangle^{n s} / R e_{\tau 0}^{2}$ decreases with increasing Reynolds number. The high level of $\left\langle R e_{\tau}^{2}\right\rangle$ over no-slip phases was demonstrated to result from the increase in momentum diffusion in $z$ direction and the spanwise gradient of $\left\langle R_{13}\right\rangle$, while $\left\langle R_{12}\right\rangle$ decreases and is thus a favorable contribution towards drag reduction.

\section{Turbulence structures}

Coherent turbulence motions can be classified into small and large scales that are responsible for dissipation and production, respectively. In this section, the changes in turbulence structures which accompany the previously examined changes in the phase-averaged statistics and skin friction are discussed. The comparisons in this section focus on the moderate and high Reynolds number cases, $R e_{b}=6785$ and $R e_{b}=10975$.

\subsection{Vortical structures}

Isosurfaces of vortical structures visualized using the $\lambda_{2}$ criterion [28] are shown in figure 13. Gray regions represent the positions of the no-slip boundary. The 

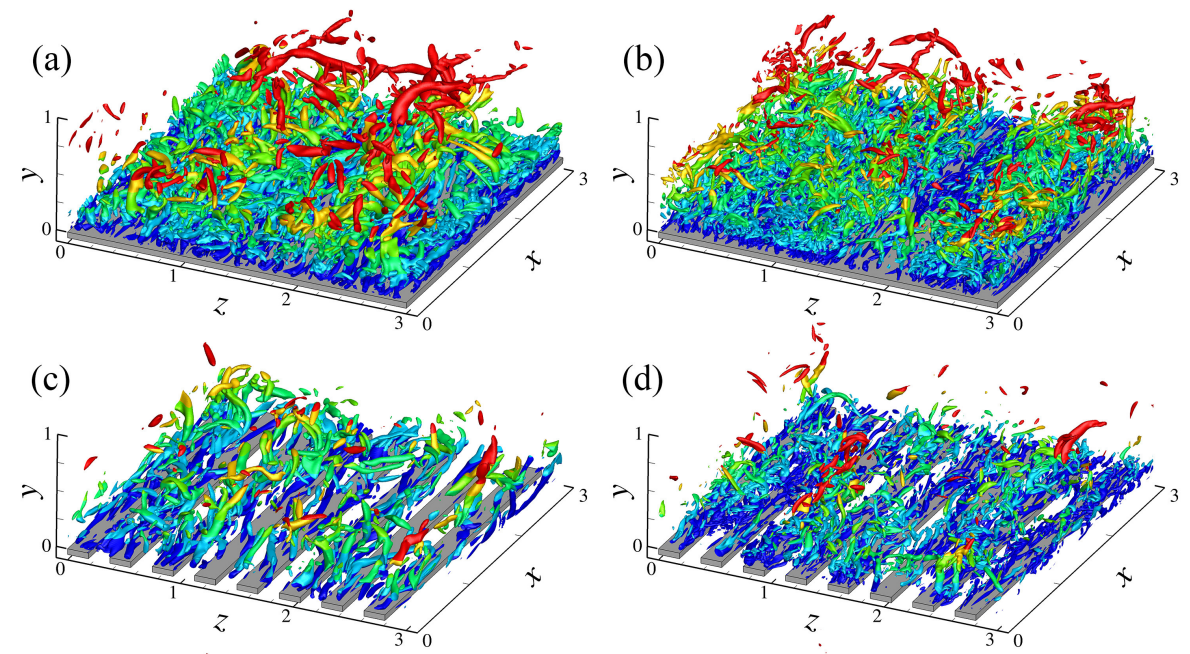

(d)
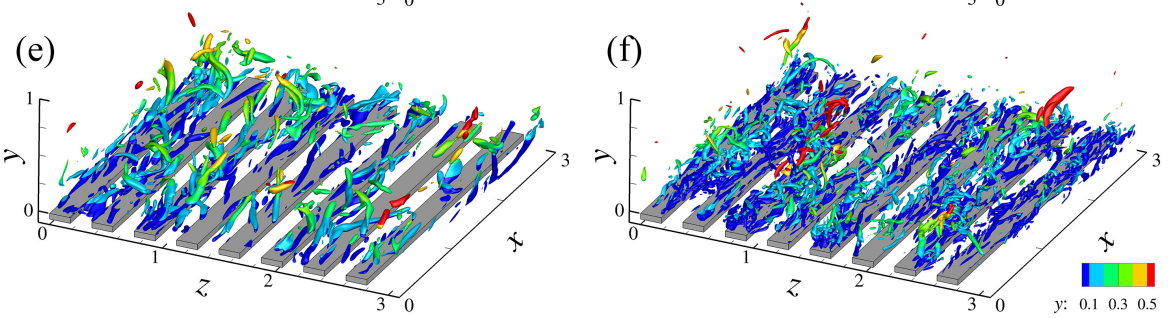

Fig. 13 Visualization of instantaneous vortical structures at (left) moderate and (right) high Reynolds number. (a-b) regular channel $\frac{\lambda_{2}}{u_{\tau 0}^{4} R e_{b}^{2}}=-0.005$; (c-d) SHS case $\frac{\lambda_{2}}{u_{\tau 0}^{4} R e_{b}^{2}}=-0.005$; (e-f) SHS case $\frac{\lambda_{2}}{\left(\left\langle u_{\tau}\right\rangle^{n s}\right)^{4} R e_{b}^{2}}=-0.005$.

presence of the SHS texture leads to a reduction in the population of vortical structures. This tendency is more evident in the low-Re flows (the snapshot of the high-Re flow is cluttered at the selected threshold, $\left.\lambda_{2} /\left(u_{\tau 0}^{4} R e_{b}^{2}\right)=-0.005\right)$. The reduction in the population of vortical structures is in line with the decrease in turbulence activity (figures 7 and 9), and is also consistent with earlier studies which reported that the strength of the vortical motions in the outer region is weakened in drag-reduced flows [21]. Note that $u_{\tau 0}$ was used for scaling of $\lambda_{2}$ in figures $13 \mathrm{c}$ and $13 \mathrm{~d}$. Other options are possible, including scaling with $\left\langle u_{\tau}\right\rangle$, $\left\langle u_{\tau}\right\rangle^{n s}$ or $\left\langle u_{\tau}\right\rangle^{x z}$. The first is not desirable since $\left\langle u_{\tau}\right\rangle=0$ on free-slip phases. 

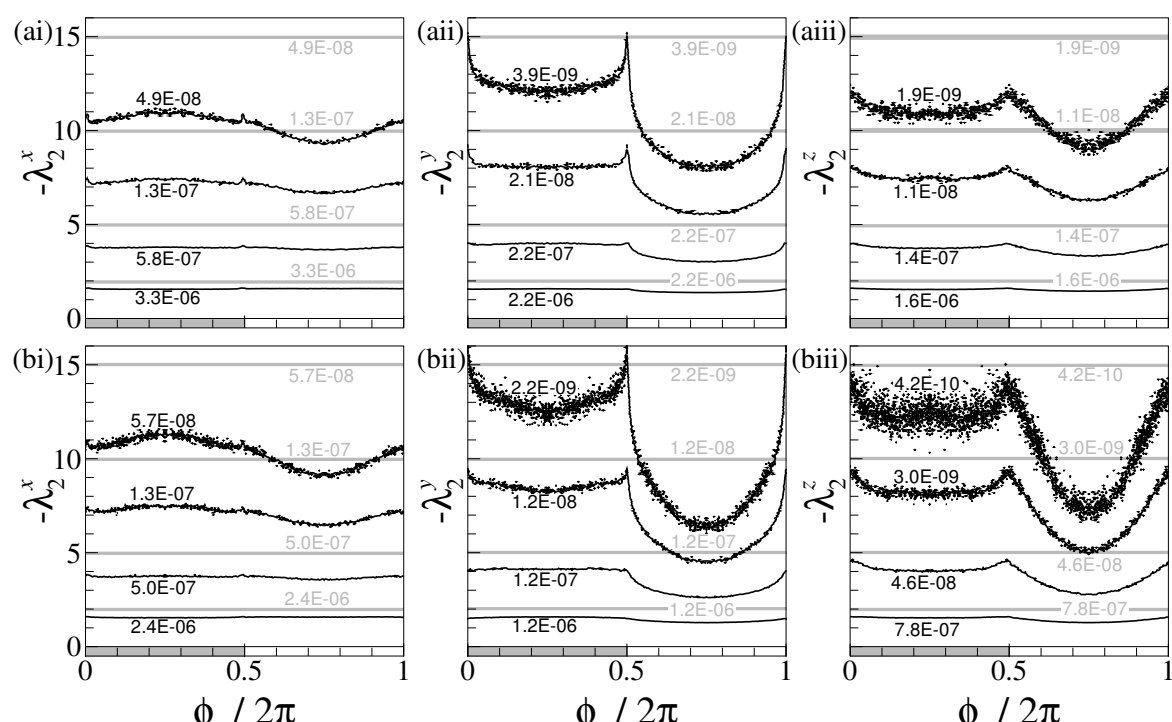

$\phi_{z} / 2 \pi$

$\phi_{z} / 2 \pi$

Fig. 14 Probability density function of the volume occupied by a particular vortical strength.

(a) M-SHS and (b) H-SHS. (i) Streamwise vortex, $-\lambda_{2}^{x}$, (ii) wall-normal vortex, $-\lambda_{2}^{y}$ and (iii) spanwise vortex, $-\lambda_{2}^{z}$. Gray contours represent the regular channel flow.

The third is feasible, but artificially alters the scaling because it includes all the free-slip phases which do not contribute to drag. The second option, $\left\langle u_{\tau}\right\rangle^{n s}$ is most relevant here since it exposes how the turbulence over the no-slip phases, where drag is generated, deviates from equilibrium wall turbulence. Isosurfaces of $\lambda_{2}$ normalized by $\left\langle u_{\tau}\right\rangle^{n s}$ also yield a scarcely populated field (figures $13 \mathrm{e}-\mathrm{f}$ ). Since the value of $\left\langle u_{\tau}\right\rangle^{n s}>u_{\tau 0}$ (c.f. figure 10), the difference between the SHS and regular channel is more pronounced when this normalization is adopted. When Re increases, although we can discern the weakening in vortical structures from instantaneous realizations (in particular red iso-surfaces in the figure), a conclusive view requires a statistical analysis.

The probability density function (pdf) of the volume occupied by $-\lambda_{2}$ was computed as a function of spanwise phase, $g\left(\lambda_{2}, \phi_{z}\right)$, and normalized to unit $\left(\lambda_{2}-\right.$ 
$\left.\phi_{z}\right)$ total area,

$$
g\left(\lambda_{2}, \phi_{z}\right) \equiv \frac{h\left(\lambda_{2}, \phi_{z}\right)}{\sum_{\lambda_{2}} \sum_{\phi_{z}} h\left(\lambda_{2}, \phi_{z}\right)}
$$

where

$$
h\left(\lambda_{2}, \phi_{z}\right) \equiv \sum_{y} \sum_{x} V\left(\lambda_{2}, \phi_{z} ; x, y\right) .
$$

In the above expression, $V$ is the volume of the computational cell that satisfies the $\lambda_{2}$ condition. Furthermore, in order to demarcate the orientation of the vortical structures, we consider the two-dimensional velocity gradient tensor [29],

$$
D_{i, j}^{2 D} \equiv\left[\begin{array}{cc}
\frac{\partial u_{i}}{\partial x_{i}} & \frac{\partial u_{i}}{\partial x_{j}} \\
\frac{\partial u_{j}}{\partial x_{i}} & \frac{\partial u_{j}}{\partial x_{j}}
\end{array}\right] .
$$

The axis of rotation of the vortical motion is then identified by a superscript, for example $\lambda_{2}^{x}(i=2$ and $j=3), \lambda_{2}^{y}(i=1$ and $j=3)$ and $\lambda_{2}^{z}(i=1$ and $j=2)$.

The pdf of $-\lambda_{2}$ is shown in figure 14. Firstly, the streamwise vortices (figure 14(i)) decrease in magnitude in the SHS flow relative to the regular channel. This trend is consistent with the study by Park et al. [14], who reported weakening of streamwise vorticity. While there is an appreciable difference between $\lambda_{2}^{x}$ of the SHS and the regular channel, the spanwise and Re dependence of $\lambda_{2}^{x}$ are not pronounced. On the other hand, the wall-normal vortical motions $\lambda_{2}^{y}$ (figure 14(ii)), display a clear $\phi_{z^{-}}$and $R e$-dependence. The wall-normal vorticity is created primarily by tilting of the mean spanwise vorticity, namely $\frac{\partial U}{\partial y}$, via the action of $\frac{\partial v^{\prime}}{\partial z}$. The sharp increase in the mean shear across the texture edge underlies the overshoot in the pdf of $\left(-\lambda_{2}^{y}\right)$ in that region. As Re increases, the difference in the pdf of the SHS and regular channels becomes more pronounced. The probability of the spanwise vortex $\lambda_{2}^{z}$ (figure 14(iii)) shows similar trends to those of $\lambda_{2}^{y}$. Because $\lambda_{2}^{z}$ is largely generated by stretching of the mean spanwise vorticity, or $\frac{\partial w^{\prime}}{\partial z}$ acting 
on $\frac{\partial U}{\partial y}$, a large peak is recorded near the texture edge. Overall however, $\lambda_{2}^{z}$ is weaker in the SHS flow relative to the regular no-slip channel and this trend is more pronounced at higher $R e$.

The presence of the SHS texture weakens the vortical motions in all three spatial directions. While the edges of the texture are hosts to an increase population of $\lambda_{2}^{y}$ and $\lambda_{2}^{z}$, the streamwise vortical structures are relatively less sensitive to the spanwise inhomogeneity of the texture. The current results on the vortical structures showed the effect of the SHS texture and the Reynolds number on the small-scale features of turbulence, especially in the outer region. These vortical structures cannot however be viewed in isolation. Changes in the large-scale coherent turbulence motions must also be examined for a more complete description of the influence of SHS textures on the flow.

\subsection{Large-scale motions}

Large-scale motion (LSM) in wall-turbulence refers to large structures which are $\mathcal{O}(\delta)$ and have coherence in the streamwise velocity fluctuation. The Reynolds shear stress $\left\langle R_{12}\right\rangle$ is primarily negative inside the LSM [30,31]. and large-scale streamwise swirls are observed at the sides of LSM [32]. In $§ 4.2 .1$, we examine the spatial characteristics of the large-scale motion and their relation to the observed changes in the shear stresses in presence of the SHS texture. 


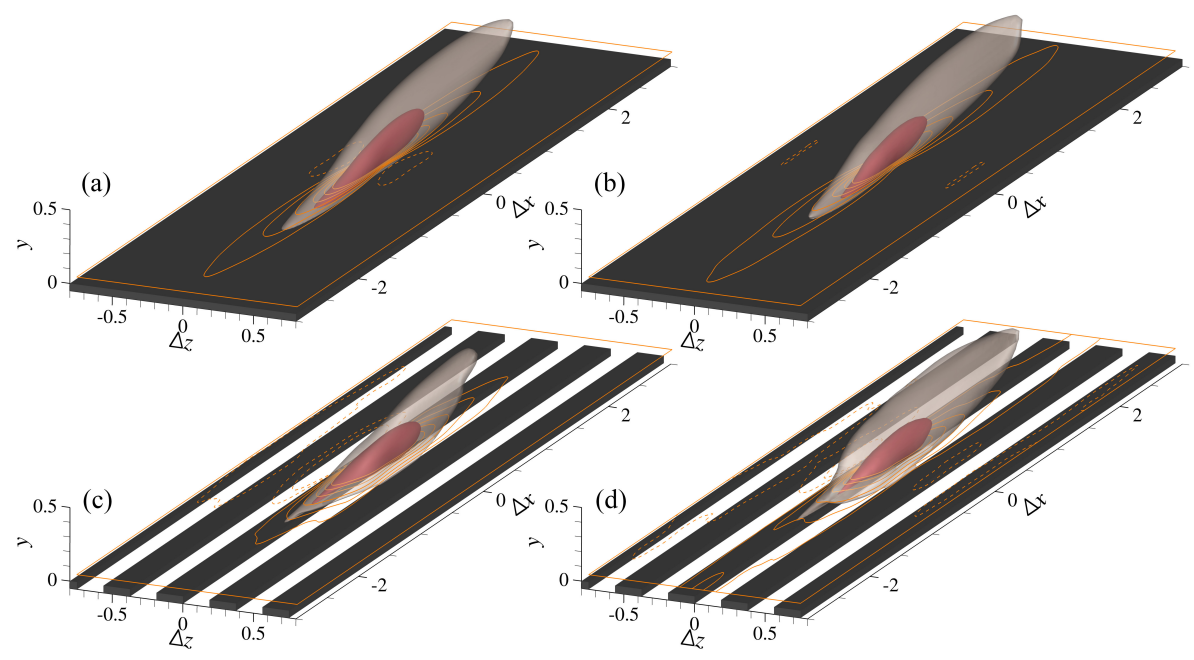

Fig. 15 Two-point correlation of streamwise velocity fluctuations, $\boldsymbol{R}_{u u}$, with $y_{\text {ref }}=0.05$ and $\phi_{z, \text { ref }}=\pi$. Iso-surfaces mark $\boldsymbol{R}_{u u}=0.3$ (red) and 0.15 (white). Line contours show an $x z$-slice at $y=y_{\text {ref. }}$ (a) M-REF, (b) H-REF, (c) M-SHS and (d) H-SHS.

\subsubsection{Auto-correlation of streamwise velocity fluctuations}

The spatial extents of large-scale motions can be evaluated using the correlation coefficient,

$$
\boldsymbol{R}_{u_{i} u_{j}}\left(\Delta x, y, \Delta \phi_{z}\right) \equiv \frac{\left\langle u_{i}^{\prime}\left(x_{\mathrm{ref}}, y_{\mathrm{ref}}, \phi_{z, \mathrm{ref}}\right) u_{j}^{\prime}\left(x_{\mathrm{ref}}+\Delta x, y, \phi_{z, \mathrm{ref}}+\Delta \phi_{z}\right)\right\rangle}{\left\langle u_{i}^{\prime}\right\rangle_{\mathrm{rms}}\left(y_{\mathrm{ref}}, \phi_{z, \mathrm{ref}}\right)\left\langle u_{j}^{\prime}\right\rangle_{\mathrm{rms}}\left(y, \phi_{z, \mathrm{ref}}+\Delta \phi_{z}\right)}
$$

In the above expression, $\left\langle u_{i}^{\prime}\right\rangle_{\mathrm{rms}}$ is the root-mean-square of $u_{i}^{\prime}$. Figure 15 shows the two-point correlation of streamwise velocity fluctuations, $\boldsymbol{R}_{u u}$. The spanwise reference position is at the NS-FS edge, $\phi_{z, \text { ref }} / 2 \pi=0.5$; the wall-normal reference position is $y_{\text {ref }}=0.05$.

The isosurface of $\boldsymbol{R}_{u u}$ over the SHS texture has a ramp shape, similar to regular no-slip channel flow. However, in line with other drag-reduced flows [21], the inclination angle (pitch in $x-y$ plane) of the isosurface decreases, although slightly. In the regular channel, the size of the correlation in the outer region is 

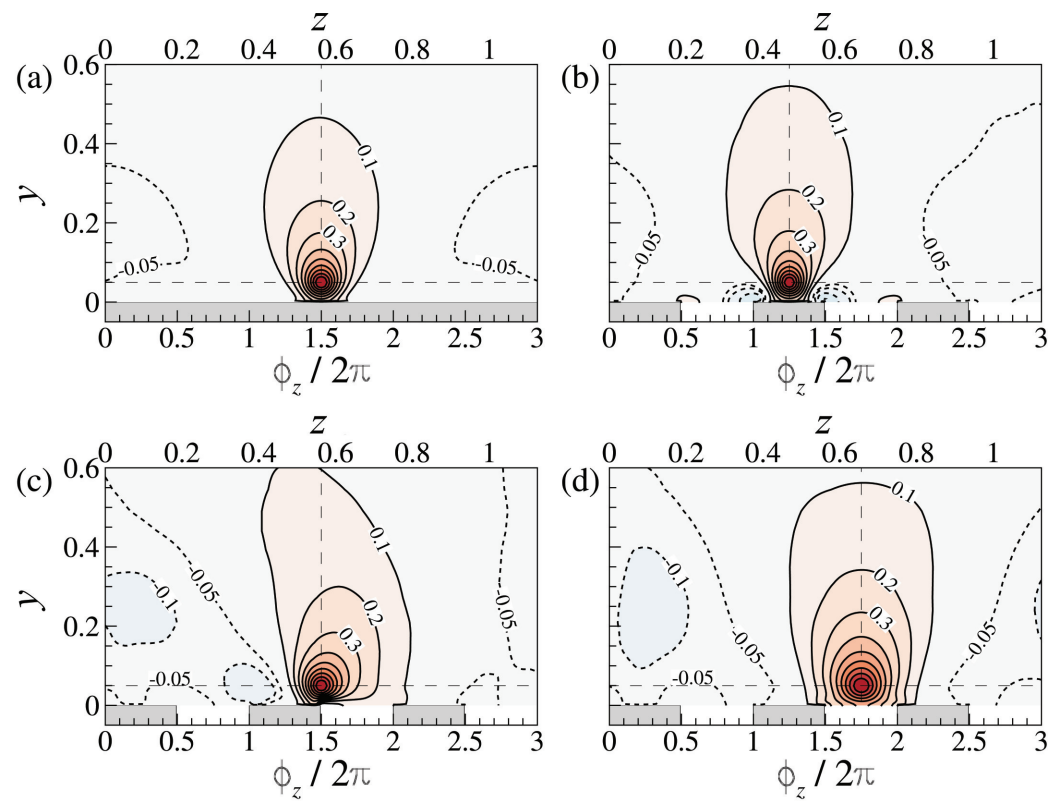

Fig. 16 Two-point correlation of streamwise velocity fluctuations, $\boldsymbol{R}_{u u}$. (a) H-REF and (b-d) H-SHS. The reference position is $y_{r e f}=0.05\left(y^{+}=29\right)$ and (b) $\phi_{z, r e f} / 2 \pi=0.25$, (c) 0.50 and (d) 0.75 in the span. Positive contours are from 0.1 to 0.9 with an increment of 0.1 . Negative contours are from -0.05 to -0.3 with an increment of 0.05 .

similar for both Reynolds numbers, which is consistent with the results by Monty et al. [33]. The SHS texture clearly affects the large-scale motions. For example, due to the presence of SHS texture and the choice of the reference position, the correlation lacks spanwise symmetry. In general, the correlation is asymmetric in the span except when the reference point is either $\phi_{z, \text { ref }} / 2 \pi=0.25$ or 0.75 .

Cross-stream views of the correlation coefficient $\boldsymbol{R}_{u u}$ at $\Delta x=0$ are shown in figure 16. This figure contains three reference positions in the spanwise direction: the center of the no-slip region $\left(\phi_{z, \text { ref }} / 2 \pi=0.25\right)$, the edge between no-slip and free-slip regions $\left(\phi_{z, \text { ref }} / 2 \pi=0.5\right)$ and the center of the free-slip zone $\left(\phi_{z, \text { ref }} / 2 \pi=\right.$ 0.75). The wall-normal reference position is $y_{\text {ref }}=0.05$, which corresponds to 
$y_{\text {ref }}^{+}=29$ for the H-SHS case. The results from regular channel flow are also included for comparison. It is evident that contours of the correlation coefficients are sensitive to the reference spanwise position. In particular, relative to the regular channel flow (figure 16a), the width of the correlation narrows over the central noslip region (figure 16b). On either side of the reference position, an anti-correlation (negative $\boldsymbol{R}_{u u}$ ) is recorded near the wall. These results indicate that, even when the reference point is on the no-slip surface, the averaged shape of large-scale motion is different in the SHS case relative to regular channel flow. When the reference point is located at the feature edge (figure 16c), the contour lines are biased towards the FS and the outer regions (towards the upper right portion of the figure, which indicates that $u^{\prime}$-events in the free-slip zone are strongly correlated with $u^{\prime}$-events in the near-wall no-slip flow. When the reference point is on the central free-slip phase (figure 16d), the correlation contours up to $\boldsymbol{R}_{u u}=0.5$ are attached to the NS-FS edge $\left(\phi_{z} / 2 \pi=1.5\right.$ and 2 in the figure $)$. The spanwise size of the largescale motion is therefore appreciably influenced by the reference $\phi_{z}$ position. It is important to note that the correlation coefficient does not provide a measure of the magnitude of $u^{\prime}$, but rather the width of the structures. We showed however that the magnitude of $\left\langle u_{i}^{\prime}\right\rangle_{\mathrm{rms}}$ is small over the NS region in figure 7 . Together, these results demonstrate that the large-scale motion over the NS region is weakened and reduced in spatial extent relative to regular channel flow.

In addition to the spanwise reference position, the Reynolds number and wallnormal reference position of the correlation significantly affect the predicted size of the structures. To evaluate these effects, figure 17 shows the maximum spanwise sizes, $l_{z}^{\text {left }}\left(\equiv z_{\text {ref }}-z_{\text {min }}\right)$ and $l_{z}^{\text {right }}\left(\equiv z_{\max }-z_{\text {ref }}\right)$, of the $\boldsymbol{R}_{u u}=0.3$ contour. When the reference point is close to the wall, $l_{z}$ depends on both the Reynolds 

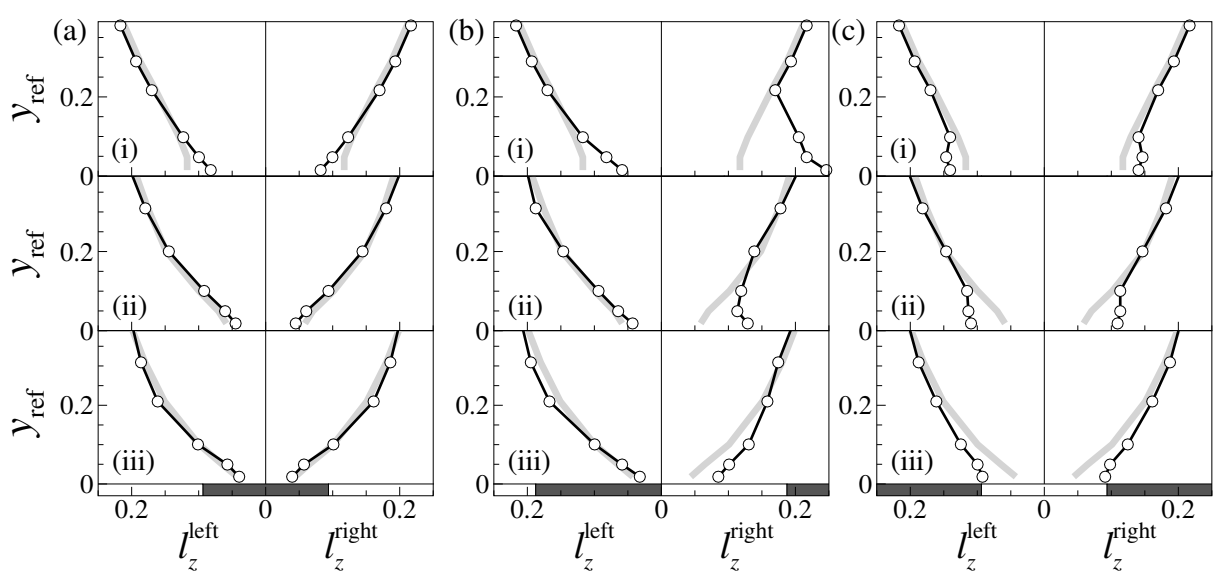

Fig. 17 Spanwise width of the $R_{u u}=0.3$ contour. (a) $\phi_{z \text {, ref }} / 2 \pi=0.25$, (b) $\phi_{z \text {, ref }} / 2 \pi=0.50$ and (c) $\phi_{z, \text { ref }} / 2 \pi=0.75$. (i) L-SHS, (ii) M-SHS and (iii) H-SHS. (Gray) regular channel flow.

number and $y_{\text {ref. }}$ On the central no-slip phase (figure 17a), the spanwise scales at small $y_{\text {ref }}$ are very slightly smaller than in a regular channel flow (gray lines in the figure, and this effect vanishes at moderate and higher $R e$ ). Figure $17 \mathrm{~b}$ shows that the correlation contours at the NS-FS edge spread towards the FS region and the deviation from the reference flow becomes more appreciable. This effect is, however, reduced at higher $R e$. The results for $\phi_{z, \text { ref }} / 2 \pi=0.75$ (figure 17c) are qualitatively similar. The trends presented here are consistent with the results from figure 11 where the spanwise influence of the texture edge was shown to diminish at larger $R e$.

\subsubsection{Conditionally-averaged velocity fields}

Low- and high-speed large-scale motions are attributed to ejection and sweep events which, in a conditional average sense, form streamwise counter-rotating rolls, akin to the secondary flow. These streamwise rolls which accompany ejection and sweep events can facilitate the transport of momentum. In the span from free- 

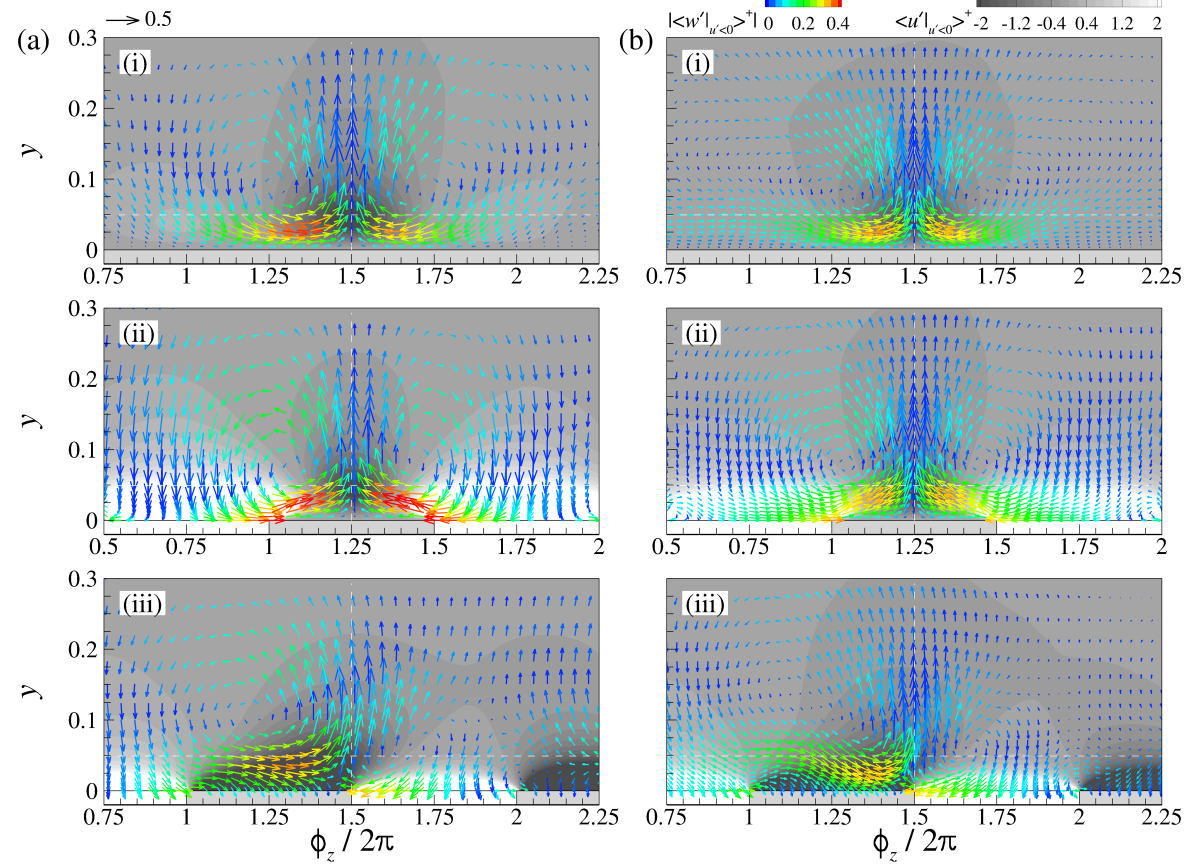

Fig. 18 Conditionally-averaged fluctuating velocity fields based on a negative- $u^{\prime}$ event in the cross-stream plane $(\Delta x=0)$. Contours show the streamwise velocity disturbance. The vectors are the in-plane velocity fluctuations, colored by the magnitude of $w^{\prime}$. (a) $R e_{b}=6785$; (b) 10975. (i) Regular no-slip channel; (ii) the SHS flows with $\phi_{z, \text { ref }} / 2 \pi=0.25$; (iii) $\phi_{z, \text { ref }} / 2 \pi=0.5$ and $y_{r e f}=0.05$.

slip onto no-slip zones, and thus enhance the mixing of mean momentum. This effect is most relevant for ejections atop a no-slip zone drawing near-wall fluid sideways from the free-slip region. Conversely, one cane consider a sweep atop a free-slip zone displacing near-wall fluid sideways onto the no-slip region.

Figure 18 shows the conditionally-averaged velocity field around negative- $u^{\prime}$ events, $\left\langle\left. u_{i}^{\prime}\left(\Delta x, y, \phi_{z}\right)\right|_{u^{\prime}\left(x, y_{\text {ref }}, \phi_{z, \text { ref }}\right)<0}\right\rangle^{+}$, which are associated with near-wall ejections. The conditional average in the regular no-slip channel flow is shown in figure 18(i) for comparison. Figure 18(ii) shows the conditionally-averaged velocity field 
when the reference position is the central no-slip phase, $\phi_{z, \text { ref }} / 2 \pi=0.25$. When the negative- $u^{\prime}$ (dark contours) are located over the no-slip region, positive- $u^{\prime}$ (light contours) are present on the free-slip portion - a feature which is qualitatively different from regular no-slip channel flow. The spanwise distance between the reference point and the cores of the streamwise rolls is reduced at high Reynolds number. While the regular channel flow has a zero spanwise velocity at the wall, the spanwise velocity in the SHS channel is finite over the free-slip region. The maximum value of $\left\langle\left. w^{\prime}\right|_{u^{\prime}<0}\right\rangle^{+}$is larger than or similar to that of the regular channel flow (see red vectors in figure 18(ii)). The position where the maximum value occurs is closer towards the texture edge, which contributes to the transport of the near-wall high-momentum fluid from the free-slip region to the no-slip region. However, the transport of the high-momentum fluid diminishes at higher Re. Recall that the two-point correlation at the texture edge was asymmetric (figure 16c). The shape of the conditional average with the reference point at the texture edge (figure 18(iii)) indicates that the swirling motion is also distorted by the texture. A small vortex forms above the free-slip region while a larger vortex appears above the no-slip zone. At this reference position, the Reynold-number effect on the maximum $\left\langle\left. w^{\prime}\right|_{u^{\prime}<0}\right\rangle^{+}$is insignificant. However, the spanwise positions of the maximum $\left\langle\left. w^{\prime}\right|_{u^{\prime}<0}\right\rangle^{+}$is more aligned with the reference position for the conditional averaging, and the size of the finite $\left\langle\left. w^{\prime}\right|_{u^{\prime}<0}\right\rangle^{+}$area (yellow and red in the figure) is smaller.

Figure 19 shows the conditionally-averaged velocity field based on a highspeed event, $\left\langle\left. u_{i}^{\prime}\left(\Delta x, y, \phi_{z}\right)\right|_{u^{\prime}\left(x, y_{\mathrm{ref}}, \phi_{z, \mathrm{ref}}\right)>0}\right\rangle^{+}$. Note that this is different to the two-point correlation which does not differentiate negative- and positive- $u^{\prime}$. Highspeed structures generally have a wide region of spanwise velocity due to the 

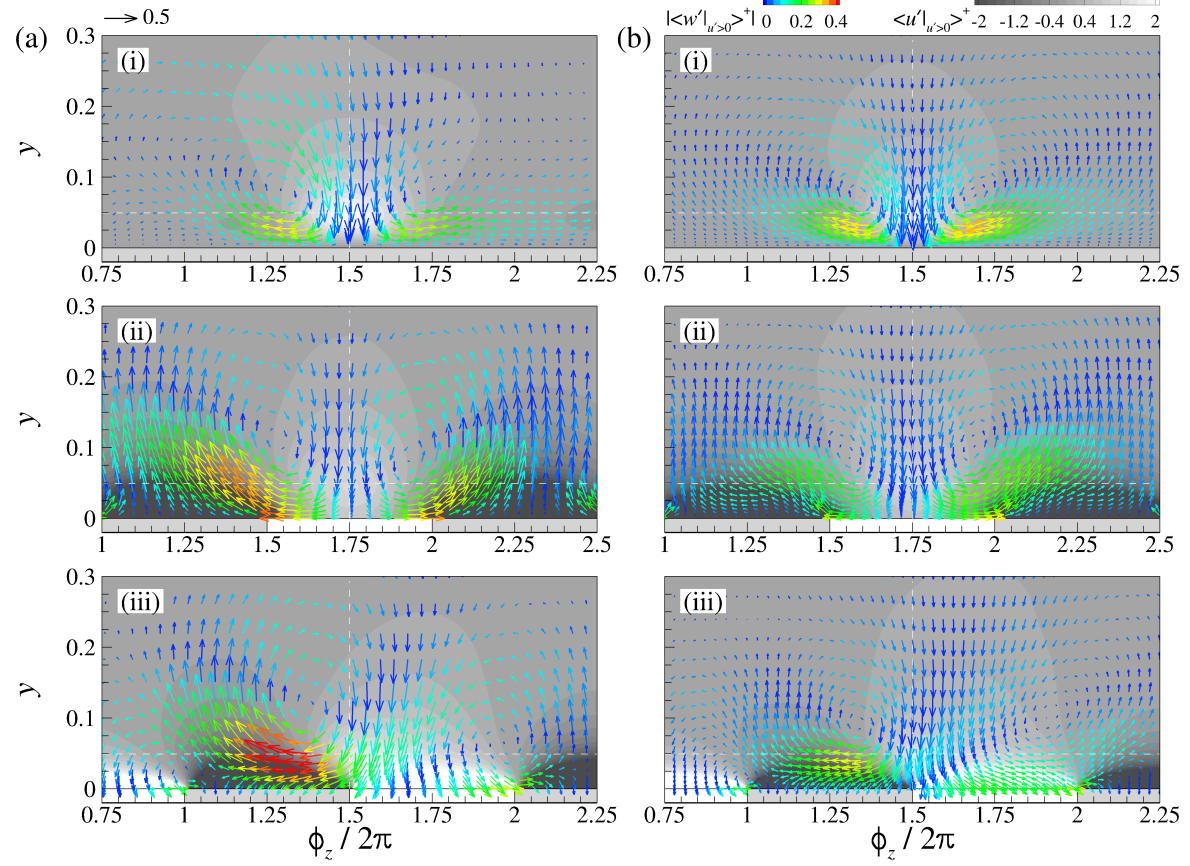

Fig. 19 Conditionally-averaged fluctuating velocity fields based on a positive- $u^{\prime}$ event in the cross-stream plane $(\Delta x=0)$. Contours show the streamwise velocity disturbance. The vectors are the in-plane velocity fluctuations, colored by the magnitude of $w^{\prime}$. (a) $R e_{b}=6785$; (b) 10975. (i) Regular no-slip channel; (ii) the SHS flows with $\phi_{z, \text { ref }} / 2 \pi=0.75$; (iii) $\phi_{z, \text { ref }} / 2 \pi=0.5$ and $y_{r e f}=0.05$.

splatting of the sweeping flow, and the width of the in-plane velocity pattern becomes smaller in outer scaling at high Re (figure 19(i)). When the reference point is the center of the free-slip region (figure 19(ii)), the sweep motion and associated near-wall spanwise motions are weakened by the SHS texture at higher Re. At low $R e$, however, the in-plane motion on the SHS texture is stronger than that of the regular channel flow. When the reference position is located at the NS-FS edge (figure 19(iii)), the positive- $u^{\prime}$ region at the reference point is connected to the free-slip phase. At high Reynolds-number, the area of positive- $u^{\prime}$ decreases 
and the in-plane vector pointing towards the no-slip phase is weakened near the wall. The present conditional averages demonstrate that the influence of stochastic fluctuations associated with ejection and sweep events at the no-slip-free-slip edge diminishes at higher Reynolds number. The overall result is a weaker spanwise momentum transport and, as a result, drag reduction approaches the gas fraction as Reynolds number is increased.

\section{Conclusions}

Fully-developed plane channel flow over streamwise-aligned superhydrphobic surface (SHS) textures was studied using direct numerical simulations at three bulk Reynolds numbers, $R e_{b}=2800,6785$ and 10975. The spanwise size of the SHS texture was identical in all cases $(d=g=0.1875 \delta)$. Regular no-slip channel flows at the same Reynolds numbers were also simulated for comparison. Previous studies reported low drag-reduction rates at relatively low $R e$ and that the dragreduction rates improves with increasing Reynolds number in superhydrophobic channels. The current study focused on explaining this trend.

The influence of the SHS texture on the flow was investigated by computing phase-averaged mean-flow and turbulence statistics and evaluating properties of the stochastic turbulence motions. The SHS texture increases the averaged streamwise velocity near the wall. All components of the Reynolds stresses are weakened in the outer flow, while there is a notable increase over the free-slip region and at the feature edges. At higher Re, the area with strong Reynolds stresses near the feature edges is reduced. Analysis of the friction Reynolds number showed that, in the no-slip region, the skin friction increases over the SHS texture. This effect 
inhibits the texture performance from reaching the ideal value, which is equal to the gas fraction, $g /(d+g)$. The improved performance at higher $R e$ is due to the changes in the mean momentum diffusion and in the turbulent diffusion in the spanwise direction. Both diffusion layers narrow at higher Re and, as a result, the performance of the texture approaches the ideal value for higher-Reynolds-number flows.

Finally, the reduction of the Reynolds shear stress and the change in the turbulent diffusion were examined and related to changes in the population of vortical structures and a weakening of the large-scale motions. Probability density functions of the small-scale vortical structures decrease in presence of the SHS texture relative to the reference no-slip channel flow. The density functions of the wallnormal and the spanwise vortices also show a pronounced spanwise dependence over the texture. Presence of the SHS texture also alters the spatial features of the large-scale motions. These structures become stretched towards the outer region of the free-slip phase, and their width in the near-wall region is confined by the narrow texture geometry. The fluctuating velocity components around the largescale motions are weaker at higher $R e$ which reduces the spanwise transport of mean momentum by coherent turbulent motions. These changes and in particular the reduction in the influence of the texture edges, all lead to the enhanced performance of the SHS at high Reynolds number.

\section{References}

1. Cassie, A., Baxter, S.: Wettability of porous surfaces. Trans. Faraday Soc., 40:546-551. 
2. Daniello, R. J., Waterhouse, N. E., Rothstein, J. P.: Drag reduction in turbulent flows over superhydrophobic surfaces. Phys. Fluids, 21:085103. (2009)

3. Wenzel, R.: Resistance of solid surfaces to wetting by water. Ind. Eng. Chem., 29:988-994. (1936)

4. Woolford, B., Prince, J., Maynes, D., Webb, B. W.: Particle image velocimetry characterization of turbulent channel flow with rib patterned superhydrophobic walls. Phys. Fluids, 21:085106. (2009)

5. Aljallis, E., Sarshar, M. A., Datla, R., Sikka, V., Jones A., Choi, C.-H.: Experimental study of skin friction drag reduction on superhydrophobic flat plates in high Reynolds number boundary layer flow Phys. Fluids, 25:025103. (2013)

6. Lee, C., Kim, C.-J.: Influence of surface hierarchy of superhydrophobic surfaces on liquid slip Langmuir, 27:4243-4248. (2011)

7. Lee, C., Kim, C.-J.: Underwater restoration and retention of gases on superhydrophobic surfaces for drag reduction Phys. Rev. Lett., 106:014502. (2011)

8. Philip, J. R.: Flows satisfying mixed no-slip and no-shear conditions. Journal of Applied Mathematics and Physics, 23:353-372. (1972)

9. Martell, M. B., Perot, J. B., Rothstein, J. P.: Direct numerical simulations of turbulent flows over superhydrophobic surfaces. J. Fluid Mech., 620:31-41. (2009)

10. Martell, M. B., Perot, J. B., Rothstein, J. P.: An analysis of superhydrophobic turbulent drag reduction mechanisims using direct numerical simulation. Phys. Fluids, 22. (2010)

11. Choi, H., Moin, P., Kim, J.: Direct numerical simulation of turbulent flow over riblets.

J. Fluid Mech, 255:503-539. (1993)

12. Türk, S., Daschiel, A., Stroh, A., Hasegawa Y., Frohnapfel, B.: Turbulent flow over superhydrophobic surface with streamwise grooves. J. Fluid Mech., 747:186-217. (2014)

13. Jelly, T. O., Jung, S. Y., Zaki, T. A.: Turbulence and skin-friction modification in channel flow with streamwise-aligned superhydrophobic surface texture. Phys. Fluids, 26:095102.

14. Park, H., Park, H., Kim, J.: A numerical study of the effects of superhydrophobic surface on the skin-friction drag in turbulent channel flow. Phys. Fluids, 25:110815. (2013)

15. Lauga, E., Stone, H.: Effective slip in pressure-driven stokes flow. J. Fluid Mech., 489:5577. (2003) 
16. Ybert, C., Barentin, C., Cottin-Bizonne, C., Joseph, P., Bocquet, L.: Achieving large slip with superhydrophobic surfaces: Scaling laws for generic geometries. Phys. Fluids, 19:123601. (2007)

17. Davis, A. M. J., Lauga, E.: Hydrodynamic friction of fakir-like superhydrophobic surfaces. J. Fluid Mech., 661:402-411. (2010)

18. Hussain, A. K. M. F., Reynolds, W. C.: The mechanisms of an organized wave in turbulent shear flow. J. Fluid Mech, 31:241-258. (1970)

19. Zaki, T. A.: From streaks to spots and on to turbulence: exploring the dynamics of boundary layer transition. Flow Turbul. Combust., 91:451-473. (2013)

20. Nolan, K. P., Zaki, T. A.: Conditional sampling of transitional boundary layers in pressure gradients. J. Fluid Mech., 728:306-339. (2013)

21. Lee, J., Jung, S. Y., Sung, H. J., Zaki, T. A.: Effect of wall heating on turbulent boundary layer with temperature-dependent viscosity. J. Fluid Mech., 726:196-225. (2013)

22. Lee, J., Jung, S. Y., Sung, H. J., Zaki, T. A.: Turbulent thermal boundary layers with temperature-dependent viscosity Int. J. Heat and Fluid Flow., 49:43-52. (2014)

23. Moser, R. D., Kim, J., Mansour, N. N.: Direct numerical simulation of turbulent channel flow up to $R e_{\tau}=590$ Phys. Fluids, 11:943-945. (1999)

24. Min, T., Kim, J.: Effect of hydrophobic surface on skin-friction drag. Phys. Fluids, 7:55-58. (2004)

25. Fukagata, K., Kasagi, N., Koumoustaskos, P.: A theoretical prediction of friction drag reduction in turbulent flow by superhydrophobic surfaces. Phys. Fluids, 18:051703. (2006)

26. Busse, A., Sandham, N. D.: Influence of an anisotropic slip-length boundary condition on turbulent channel flow. Phys. Fluids, 24:055111. (2012)

27. Fukagata, K., Iawmoto, K., Kasagi, N.: Contribution of Reynolds stress distribution to the skin friction in wall-bounded flows. Phys. Fluids, 14:73-76. (2002)

28. Jeong, J., Hussain, F.: On the identification of a vortex. J. Fluid Mech., 285:69-94. (2009)

29. Adrian, R. J., Christensen, K. T., Liu, Z. C.: Analysis and interpretation of instantaneous turbulent velocity fields. Exp. Fluids, 29:275-290. (2000a)

30. Adrian, R. J., Meinhart, C. D., Tomkins, C. D.: Vortex organization in the outer region of the turbulent boundary layer. J. Fluid Mech., 422:1-54. (2000b) 
31. Ganapathisubramani, B., Longmire, E. K., Marusic, I.: Characteristics of vortex packets in turbulent boundary layers. J. Fluid Mech., 478:35-46. (2003)

32. Hutchins, N., Hambleton, W. T., Marusic, I.: Inclined cross-stream stereo particle image velocimetry measurements in turbulent boundary layers. J. Fluid Mech., 541:21-54. (2005)

33. Monty, J. P., Stewart, J. A., Williams, R. C., Chong, M. S.: Large-scale features in turbulent pipe and channel flows. J. Fluid Mech., 589:147-156. (2007) 